\title{
Neurite Sprouting and Synapse Deterioration in the Aging Caenorhabditis elegans Nervous System
}

\author{
Marton Lorant Toth, ${ }^{1}$ Ilija Melentijevic, ${ }^{1}$ Leena Shah, ${ }^{1}$ Aatish Bhatia, ${ }^{1}$ Kevin Lu, ${ }^{1}$ Amish Talwar, ${ }^{1}$ Haaris Naji, ${ }^{1}$ \\ Carolina Ibanez-Ventoso, ${ }^{1}$ Piya Ghose, ${ }^{2}$ Angela Jevince, ${ }^{3}$ Jian Xue, ${ }^{1}$ Laura A. Herndon, ${ }^{3}$ Gyan Bhanot, ${ }^{1}$ Chris Rongo, ${ }^{2}$ \\ David H. Hall, ${ }^{3}$ and Monica Driscoll ${ }^{1}$ \\ ${ }^{1}$ Department of Molecular Biology and Biochemistry, Nelson Biological Laboratories, Rutgers, The State University of New Jersey, Piscataway, New Jersey \\ 08854, ${ }^{2}$ Department of Genetics, Waksman Institute, Rutgers, The State University of New Jersey, Piscataway, New Jersey 08854 , and ${ }^{3}$ Center for C. elegans \\ Anatomy, Albert Einstein College of Medicine, Bronx, New York, 10461
}

Caenorhabditis elegans is a powerful model for analysis of the conserved mechanisms that modulate healthy aging. In the aging nematode nervous system, neuronal death and/or detectable loss of processes are not readily apparent, but because dendrite restructuring and loss of synaptic integrity are hypothesized to contribute to human brain decline and dysfunction, we combined fluorescence microscopy and electron microscopy (EM) to screen at high resolution for nervous system changes. We report two major components of morphological change in the aging C. elegans nervous system: (1) accumulation of novel outgrowths from specific neurons, and (2) physical decline in synaptic integrity. Novel outgrowth phenotypes, including branching from the main dendrite or new growth from somata, appear at a high frequency in some aging neurons, but not all. Mitochondria are often associated with age-associated branch sites. Lowered insulin signaling confers some maintenance of ALM and PLM neuron structural integrity into old age, and both DAF-16/FOXO and heat shock factor transcription factor HSF-1 exert neuroprotective functions. $h s f-1$ can act cell autonomously in this capacity. EM evaluation in synapse-rich regions reveals a striking decline in synaptic vesicle numbers and a diminution of presynaptic density size. Interestingly, old animals that maintain locomotory prowess exhibit less synaptic decline than same-age decrepit animals, suggesting that synaptic integrity correlates with locomotory healthspan. Our data reveal similarities between the aging C. elegans nervous system and mammalian brain, suggesting conserved neuronal responses to age. Dissection of neuronal aging mechanisms in C. elegans may thus influence the development of brain healthspan-extending therapies.

\section{Introduction}

Aging of the human brain, contributing to disease and dysfunction, has emerged as a major health issue of our time. Healthy brain aging is characterized by subtle refinements such as synaptic deterioration, neuronal sprouting, and restructuring, rather than neuronal loss (Yankner et al., 2008). These physical changes have been suggested to underlie cognitive decline (Yankner et al., 2008; Bishop et al., 2010; Fjell and Walhovd, 2010) and may promote neuronal dysfunction in neurodegenerative diseases (Knobloch and Mansuy, 2008). Still, the physiological factors that influence structural neuronal decline over time within native

Received March 23, 2011; revised March 8, 2012; accepted March 20, 2012.

Author contributions: M.L.T., D.H.H., and M.D. designed research; M.L.T., I.M., L.S., K.L., A.T., H.N., A.J., and D.H.H. performed research; M.L.T., C.I.-V., P.G., L.A.H., C.R., D.H.H., and J.X. contributed unpublished reagents/ analytic tools; M.L.T., A.B., K.L., H.N., G.B., and D.H.H. analyzed data; M.L.T., A.B., D.H.H., and M.D. wrote the paper.

This work was supported by grants from the New Jersey Commission on Spinal Cord Research (M.T. and M.D.) and the National Institutes of Health (AG024882; NS034435, M.D.; 0D010943; RFK-IDDRC Grant HD071593, D.H.H.). M.T.T. is currently supported by an American Federation of Aging Research Postdoctoral Fellowship. We thank the Caenorhabditis Genetic Center, supported by NIH NCRR, for providing strains. We also thank S. Shaham for reagents, B. Grant, M. Barr, and E. Vayndorf for critical reading of this manuscript and N. Hall for statistical help for synaptic vesicle data analysis.

Correspondence should be addressed to Monica Driscoll, Department of Molecular Biology and Biochemistry, Rutgers, The State University of New Jersey, 604 Allison Road, Nelson Biological Laboratories A232, Piscataway, NJ 08854. E-mail:driscoll@biology.rutgers.edu.

DOI:10.1523/JNEUROSCI.1494-11.2012

Copyright $\odot 2012$ the authors $\quad 0270-6474 / 12 / 328778-13 \$ 15.00 / 0$ context remain poorly understood, especially at the single cell level.

The nematode Caenorhabditis elegans has proven to be a powerful model for the study of aging biology. The 959-celled transparent $C$. elegans lives $\sim 3$ weeks, and genetic manipulations have identified hundreds of genes that can influence longevity, implicating conserved signaling pathways, such as insulin/IGF-like signaling, in lifespan control (Antebi, 2007; Kenyon, 2010). Interestingly, C. elegans tissues deteriorate at different rates in aging adults, with certain features of ageassociated decline strikingly reminiscent of those in higher organisms (Garigan et al., 2002; Herndon et al., 2002; Glenn et al., 2004). For example, like humans, aging C. elegans experience a decline in muscle mass, structure, and strength (sarcopenia) occurring with mid-life onset (Herndon et al., 2002; Glenn et al., 2004). Our previous characterization of the aging C. elegans nervous system suggested that, like in aging human brain, neurons do not die and axons do not disintegrate (Herndon et al., 2002). At the same time, several studies have established that $C$. elegans neurons are important in influencing organismal longevity: genetically lowering insulin/IGF signaling only in neurons can extend lifespan (Wolkow et al., 2000; Iser et al., 2007), as can elimination of neuronal function (Apfeld and Kenyon, 1999) or of specific neurons themselves (Alcedo and Kenyon, 2004). Complex neuroendocrine effects exert tremendous impact on the ag- 
ing of the animal (Panowski and Dillin, 2009). Given these data and the interest in the subcellular changes that underlie ageassociated dysfunction in mammalian nervous system aging, we examined the structure of the aging C. elegans nervous system using fluorescence and electron microscopy.

Here, we report two striking features of the aging C. elegans nervous system-structural regrowth and synaptic deterioration. Morphological changes that occur during aging are neuron specific and include new dendrite outgrowth from processes or somata, with mitochondria often situated at the branch point for the new neurite. Regrowth abnormalities can be deferred in part by insulin receptor daf-2 reduction-of-function (rf), supporting that neuronal healthspan is regulated by the insulin signaling pathway. Downstream transcription factors DAF-16/FOXO and HSF-1 are neuroprotective against structural aging of touch receptor neurons. Synaptic decline features a decrease in presynaptic terminal size and a reduction in numbers of synaptic vesicles close to the synapse, suggestive of a decline of robustness in synaptic signaling. Since morphological branching/sprouting and synaptic decline in the absence of cell death typifies aging human brain, key factors in age-associated neuronal decline may be conserved across phyla.

\section{Materials and Methods}

C. elegans strains and genetics

C. elegans strains were grown on nematode growth media (NGM) plates streaked with live Escherichia coli OP50-1 (a streptomycin-resistant derivative of OP50) at $20^{\circ} \mathrm{C}$ as described previously (Brenner, 1974). Wild-type worms were C. elegans var. Bristol (N2). Other strains were as follows: ZB154 zdIs5[pmec-4::gfp], KWN176 rnyIs014[pmec-4::mcherry; unc-119(+)], BZ555 egIs1[pdat-1::gfp], EG1285 oxIs12 [unc-47p::GFP + lin-15(+)], NC1750 gvEx173[opt-3::gfp + pRF4(rol-6)], CB1370 daf-2(e1370), DR1572 daf-2(e1368), GR1307 daf-16(mgDf50), PS3551 hsf-1(sy441), OR2451

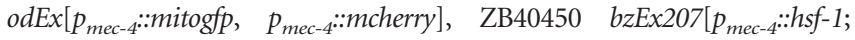
$\left.p_{m e c-4}:: m c h e r r y\right] ; ~ h s f-1(s y 441)$. The daf-16(mgDf50) allele was tracked by PCR amplification of genomic sequence encompassing the deletion with specific primers (5'TTCAGTCCCCGAACTCAATC3' and 5'GGAATTGT AGCTGCACAGCA3 $\left.{ }^{\prime}\right)$. OR2451 was made by coinjecting $p_{\text {mec }-4}:$ mitogfp $(10$

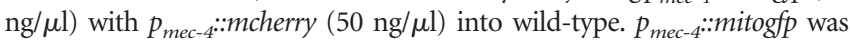
generated by introducing the mec-4 promoter (from $z d I s 5$ ) ligated to the

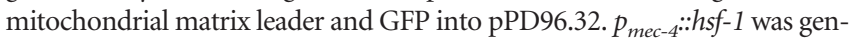
erated by replacing the $o s m-6$ promoter in front of the $h s f-1$ transgene in plasmid pTB34 (Bacaj and Shaham, 2007) with the mec-4 promoter using primers GCTCTAGAGCCAATACAAGCTCAAATAC and TCCCCC CGGGGGGACTCTATAACTTGATAGCGATAAAAAAAAT to amplify

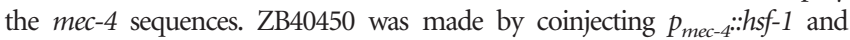
$p_{\text {mec-4: }}:$ mcherry $(100 \mathrm{ng} / \mu \mathrm{l}$ each) into wild type and then crossing the resulting transgene array $b z E x 207$ into $h s f-1$ (sy441).

\section{Neuronal aging assays}

C. elegans strains were grown at $20^{\circ} \mathrm{C}$ for at least two generations before the experiments. For synchronization, 20-30 gravid well fed adults (P) were transferred to a new NGM plate with live E. coli OP50-1 bacteria to lay eggs for $4-5 \mathrm{~h}$, and then removed. F1 hermaphrodite animals were maintained at $20^{\circ} \mathrm{C}$ until the late $\mathrm{L} 4$ larval stage (characterized by the appearance of the "Christmas tree vulva") then transferred to the final assay plates (1 worm/plate) supplemented with $51 \mu \mathrm{M}$ FUDR (5-fluoro2 '-deoxyuridine, Sigma, F0503) and UV killed OP50-1 bacteria and scored at $24^{\circ} \mathrm{C}(t=0$, day 1$)$. Control experiments $+/ 1$ FUDR confirmed that ALM and PLM abnormalities were not changed by drug; a slight increase in AVM and PVM abnormalities was observed with FUDR, but these changes remained rare. FUDR was not used for studies shown in Figures $4 J, 5,6$, tests of daf-2(e1368) not shown, and quantitative analysis of touch neuron mitochondria (see Fig. $3 G$, legend). In other studies, we focused all our major quantitative analysis on ALM and PLM abnormalities, phenotypes not influenced by FUDR.
Animal age throughout the paper refers to the adult age measured in days, calculated by subtracting the date when the given animal went through the L4-A molt from the date of examination. Animals were considered dead when they stopped pharyngeal pumping and failed to respond to touch by an eyelash. Two methods were used to monitor aging neurons. In the first method (results in Fig. 5), 12 animals were serially measured on adult days 2, 4, 6, 8. Animals were transferred to $10 \mathrm{~mm}$ levamisole solution (Sigma-Aldrich, L9756) on Neuronal Aging slides, made of two cover glasses (Fisher Scientific, 22-050-246) and custom image spacers (Grace Bio, 1L44010), then scored on a Zeiss Imager D1m upright compound microscope. In the second method, animals were scored on a Kramer M2 Bio Quad microscope (36 per measurement trial; results in Figs. 1-4). Behavioral touch assay using a fine $0.025 \mathrm{~mm}$ thin platinum wire (Alfa Aesar, AA10292H4) was performed before monitoring the neuronal aging phenotypes. Each experiment was done in triplicate and results were compared with a control strain carrying only the transgene that labels the respective neurons, prepared, maintained, and monitored strictly in parallel with the test strain.

\section{Data management}

Neuronal aging phenotypes were recorded in online spreadsheets (Google), then downloaded and converted by the Neuronal Aging Data Converter Program. Converted files were analyzed by the Neuronal Aging Statistics Package for Python, diagrams were created using Microsoft Excel, 2008 for Mac (Microsoft Corporation).

\section{Statistical analysis}

Mean phenotype occurrences and probabilities of a neuron being altered were calculated using the Enthought Python Distribution (7.0-1 for $\mathrm{Mac})$. All comparisons of mean occurrences were done using an Independent $t$ test; probability of altered cells was calculated using the twotailed Fisher's exact test (Fisher 0.1.4 package for Python). Survival curves were produced using the Kaplan-Meier method and compared using the log rank test. SD of the mean lifespan for Kaplan-Meier Estimator was calculated using Greenwood's formula. Error bars in all figures indicate the SD of the samples. Asterisks show significant difference $(p<0.05)$.

Within each experiment for all the different neurons, for each day, and for each genotype, we compared the above statistics in the following ways: age-based comparisons (comparisons between different days), left versus right comparisons (comparisons between $\mathrm{L}$ and $\mathrm{R}$ neurons), touch sensitivity comparisons (comparison between cells from worms in touch sensitivity class $\mathrm{AP}+\mathrm{A}$ vs 0 for anterior assay, $\mathrm{AP}+\mathrm{P}$ vs 0 for posterior assay data in Figs. 1, 2, 4), and survival statistics (mean lifespan with SD, lifespan differences between strains). For characterization of the basic neuronal aging phenotypes we pooled selected control measurements and performed the statistical analysis on this large sample. To estimate the effects of daf-2, daf-16, and $h s f-1$ gene inactivation/expression on neuronal aging we only used data from the mutants and their respective wild-type controls from that specific experiment to ensure same condition comparisons.

\section{Electron microscopy}

C. elegans wild-type animals were raised at $20^{\circ} \mathrm{C}$ to create synchronized cultures and maintained at $20^{\circ} \mathrm{C}$ throughout their lives. Adults were raised on NGM plates with live E. coli OP50-1 and transferred every 2-3 $\mathrm{d}$ while they were reproductive. At $15 \mathrm{~d}$ of adulthood, animals were fixed and embedded for TEM following a standard chemical immersion protocol (Hall, 1995). Thin sections were collected onto formvar-coated slot grids, poststained with uranyl acetate and lead citrate, and examined using a Philips CM10 electron microscope. Images were collected on Kodak 4489 film or using an Olympus Morada digital camera, and figures were produced using iTEM and Photoshop software.

\section{Electron microscopic evaluation of aging neurons}

Measurement of synapse dimensions. Synapses were compared mostly from the nerve ring and ventral ganglion in several animals per treatment group that were all fixed by similar methods (Hall, 1995). The identities of the cells making particular synapses were not discerned. In previous 

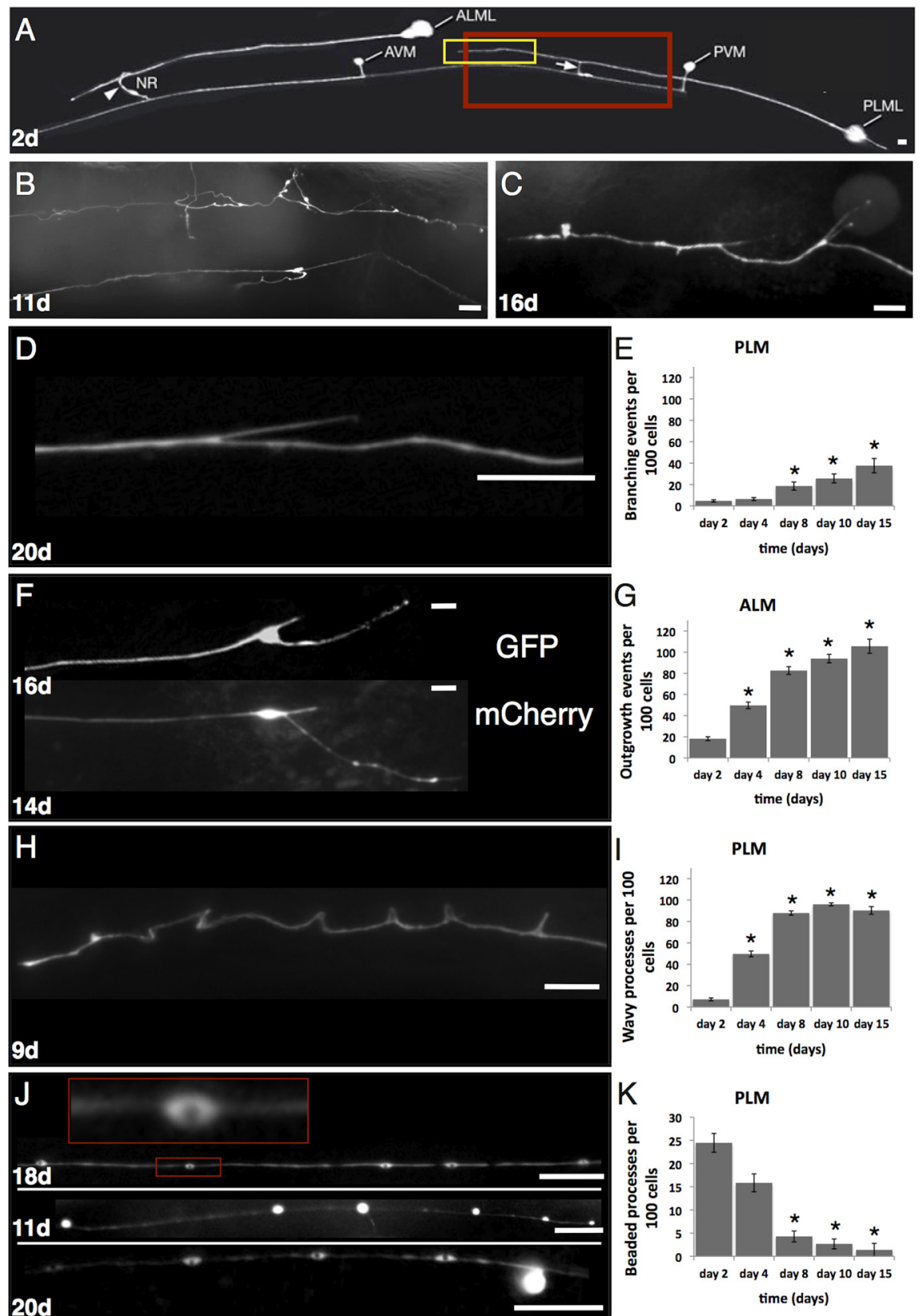

Figure 1. C. elegans touch receptor neurons exhibit morphological changes with age. A, GFP-visualized touch receptor neurons

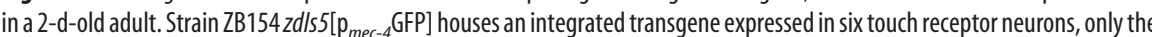
left anterior ALM and posterior PLM are visible in this focal plane. Native branches at distal ends of ALM (arrowhead) and PLM (arrow) are indicated. Highlighted boxes indicate the regions analogous to those magnified in $\boldsymbol{B}$. Note the uniform distribution of GFP along the processes and the short posterior extension from the PLM soma typical for young adults. Panel is taken from www.wormatlas.org. $\boldsymbol{B}, \boldsymbol{C}$, Examples of dramatic morphological changes that can occur in aging touch neurons. $\boldsymbol{B}$, Magnified

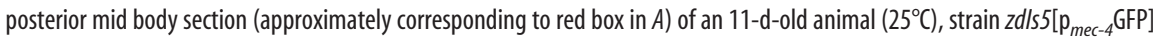
PLMR is the top neuron, PVM is the bottom neuron. $C$, Magnified body section (approximately corresponding to yellow box in $A$ ) in a 16-d-old PLML $\left(25^{\circ} \mathrm{C}\right)$, strain $z d / s 5\left[\mathrm{p}_{\text {mec- }-4} \mathrm{GFP}\right]$. Neuronal processes exhibit novel branches, wavy appearance, and some GFPbeading. $\boldsymbol{D}-\boldsymbol{I}$, Examples and quantitation of morphological changes in aging touch receptor neurons. For all experiments, day 1 is the beginning of adulthood $\left(24^{\circ} \mathrm{C}\right)$ and animals were scored only on the day indicated, rather than sequentially. Numbers of neurons of indicated types scored per time point were as follows: 428 day $2 ; 340$ day $4 ; 280$ day $8 ; 224$ day $10 ; 72$ day 15 . Unless otherwise stated, photographs are of strain ZB154 zdls5[ $\left.\mathrm{p}_{\text {mec-4 }} \mathrm{GFP}\right]$, anterior is to the left. $\boldsymbol{D}$, Typical branched process phenotype in an aging PLM neuron, $20 \mathrm{~d}$-old. $\boldsymbol{E}$, Quantitation of novel dendrite branching phenotypes in PLM neurons in aging adults. Total numbers of major novel branches observed/ 100 neurons are indicated. We counted branches across the entire anteriorly projecting process, excluding the normal developmental branch. We scored at $600 \times$ magnification using a dissecting microscope for relatively high throughput scoring up to day 10 . Small novel branches would have been missed at this magnification, so the rate of branch occurrence presented is a likely underestimate. At day 15 , gut autofluorescence often precluded observation of branching in the dissecting microscope, so we scored at $600 \times$ on a compound scope with a higher numerical aperture objective $(10 \times)$ to limit background fluorescence. Tiny novel branches visualized with the compound microscope at day 15 were not included in the mutant studies affecting vesicle recycling at chemical synapses, we observed that the shape and size of individual synapses is quite flexible, and that synapses holding smaller numbers of vesicles generally have smaller diameters (Lesa et al., 2003; Marza et al., 2008; Govorunova et al., 2010). The appearance of the presynaptic bar is variable in normal and aging synapses, depending on section angle. The bar is usually longer than it is wide, and usually can be seen across multiple thin sections (White et al., 1986). Measurements were made in isolated single thin sections, with the appreciation that the section angle limits the ability to accurately determine the exact synapse dimensions in both young and old synapses.

\section{$\leftarrow$}

statistical analysis, so $15 \mathrm{~d}$ data count only large branch scores similar to those detected with the dissecting microscope. Asterisk indicates $p<0.05$ for independent $t$ test, comparison to day 2 adult, error bars indicate the SD. $\boldsymbol{F}$, Typical soma outgrowth phenotypes in ALM neurons. Top, $z d l s 5\left[\mathrm{p}_{\text {mec- } 4} \mathrm{GFP}\right]$, $16 \mathrm{~d}$ old; bottom, strain KWN177, rnyls014[p $\mathrm{p}_{\text {mec }-4}$ mCherry; unc-119(+)], 14 d old. G, Quantitation of soma outgrowths in aging ALM neurons. In young adults, we find only $18 \%$ of soma exhibit the small posterior extension in $2 \mathrm{~d}$-old adults. Because of the relatively infrequent occurrence of this feature, we counted the presence of any extension as one outgrowth event. Total numbers of outgrowths per 100 cell observations are indicated. Some soma-anchored processes can grow substantially with increasing age (although growth appears to stop several days before death), but we have not quantitatively scored the total amount of growth. Microscopy protocol for observation was as described for $\boldsymbol{E}$; statistics as in $\boldsymbol{E}$. $\boldsymbol{H}$, Example of a PLM neuron of $z d / 55\left[p_{\text {mec- } 4}\right.$ GFP] in which the process appears wavy and exhibits some acute bends, $9 \mathrm{~d}$ old. I, Quantitation of wavy process occurrence in aging PLM neurons. We scored a process as wavy if it appeared wavy or bent at any place along the process (in contrast to the normal straight trajectory); waves or bends remained in place in a moving animal, so appear to be fixed characteristics of the neuron rather than dependent upon the posture of the animal. Scoring microscopy was as described for $\boldsymbol{D}$. Error bars indicate $S D$, statistics as in $\boldsymbol{E}$. J, Examples of bead-like GFP accumulations in PLM processes. Top, GFP accumulates with a beaded appearance with central regions of GFP exclusion - inset, enlarged view of a "bead" with a dark, GFP-free center (18 d old). Middle, Uniform bright accumulations that sometimes appear to extend off the main process as protrusions ( $11 \mathrm{~d}$ old). Bottom, A process with both beaded structures and a rounded extension protruding from the right end of the process ( $20 \mathrm{~d}$ old). Beads are unlikely to correspond to novel synaptic structures on the normally synapse-free sensory process as the synaptic protein reporter SNB-1::GFP does not concentrate at these beads to levels observed at synapses and this SNB-1::GFP faint signal can still be observed in the absence of beads/protrusions; faint SNB-1::GFP signals can be found in $\sim 1 / 3$ of transient beads that move as if being trafficked (data not shown). Other work has shown that similar beads/protrusions can include disorganized microtubules and do not correlate with a lysosomal marker (Pan et al., 2011). $\boldsymbol{K}$, Quantitation of the occurrence of beaded processes of aging PLM neurons. We scored a process as containing GFP beads or protrusions if there were $>10$ GFP accumulations per process; both uniformly filled and central GFP exclusion beads were counted together. Microscopy scoring was as described for $\boldsymbol{D}$. Error bars indicate SD, statistics as in E. Scale bars: $A-J: 10 \mu \mathrm{m}$. 


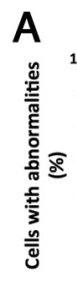

A
AVM

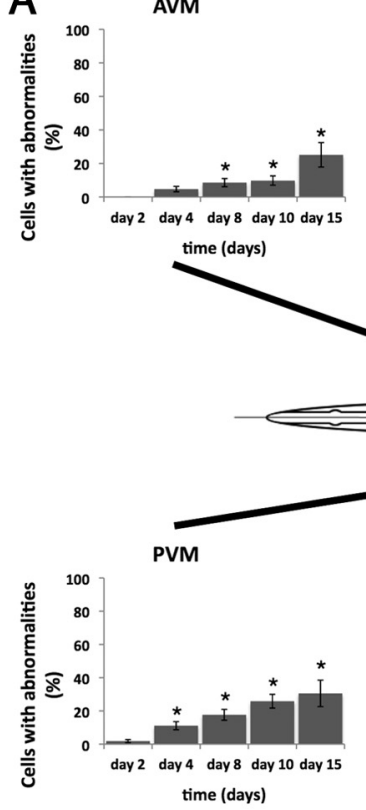

B

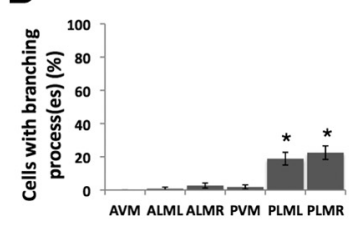

time (days)
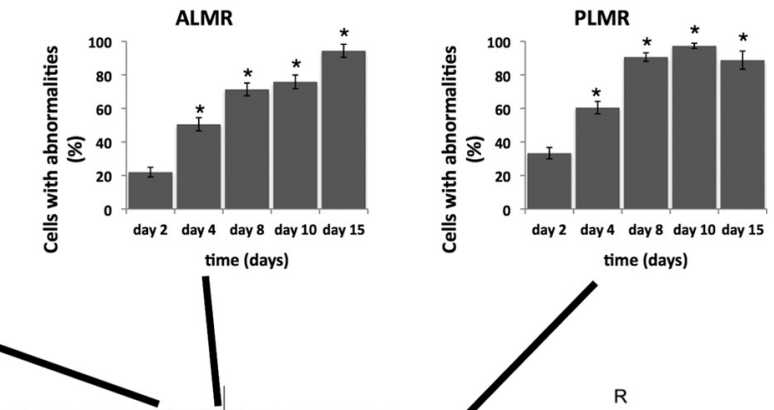

time (days)
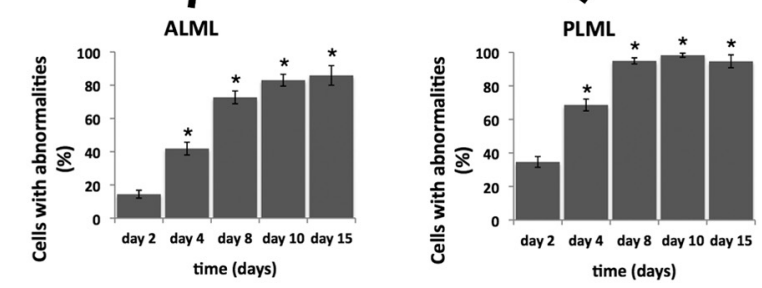

Figure 2. Morphological abnormalities in touch neurons increase with age, with the specific abnormality type differing markedly among touch neuron subtypes. $A$, The summed incidence of common abnormalities increases with age in all touch neurons, but not all touch neurons exhibit similar aging profiles. We graphed the percentage of each touch neuron (ALML/R, PLML/R, AVM, PVM) that exhibited any of the three common morphological abnormalities (branched process, soma outgrowth, wavy processes) on the days indicated, $24^{\circ}$. Error bars are for $S D^{*}{ }^{*} p<0.05$, Fisher's exact test, compared with day 2 . Touch receptor soma positions are indicated by dots in C. elegans graphic; process lengths scored are similar for all touch neurons except for PVM, which was evaluated only until it runs into the ventral nerve cord $(\sim 15 \times$ shorter than PLM process length examined). Numbers of neurons of each type scored per time point were as follows: 214 day 2; 170 day $4 ; 140$ day $8 ; 112$ day $10 ; 36$ day 15 . Note that although all touch neurons expressed an age-associated increase in morphological abnormalities, not all touch neurons exhibited similar aging profiles. $\boldsymbol{B}$, Different touch receptor neurons exhibit different morphological abnormality types as they age. Compared are the percentage of neurons of the indicated types that exhibited a branched process (left) soma outgrowth (middle), or wavy processes (right). One-hundred neurons were scored for each type, day 10 of adult life at $24^{\circ} \mathrm{C}$, evaluation protocol as described for Figure $1 E$, numbers of neurons scored as in $\boldsymbol{A}$; error bars indicate SD. Note striking differences in frequencies of the different abnormality types observed in ALMs versus PLMs versus AVM/PVM. Although the frequencies of abnormalities are lower in AVM and PVM, these neurons differ from each other in soma outgrowth and wavy process formation frequencies. ${ }^{*} p<0.05$, Fisher's exact test, comparing to AVM scores for the indicated abnormality. We documented cell-specific comparisons at additional time points during adult life and find similar trends on other days (data not shown). Studies presented were performed in the presence of $51 \mu \mathrm{g} / \mathrm{ml}$ FUDR in plates to prevent progeny production. We found no statistical difference in ALM and PLM abnormalities if we used $51 \mu \mathrm{g} / \mathrm{ml} \mathrm{FUDR} \mathrm{versus} \mathrm{animals} \mathrm{not} \mathrm{reared} \mathrm{on} \mathrm{the} \mathrm{drug} \mathrm{but} \mathrm{picked}$ away from offspring (data not shown); we did note a slight increase in abnormalities in AVM and PMV at this concentration FUDR, underscoring cell type differences. These changes remained rare, however.

Vesicle counts in aging neurons. In aging neurons, electron density of the cytoplasm is variably affected and can become excessively dark, especially near the presynaptic bar. This factor interfered with vesicle counting in some cases, so that our "counts" are sometimes approximated. In order not to ignore synapses that are severely depleted due to aging, we estimated counts even for rather degenerated synapses. Despite the wide range of synapse phenotypes in both wild-type and aging animals, the trends we report are striking, highly prevalent, and hold statistical significance.

\section{Results}

\section{C. elegans touch receptor neurons exhibit new neurite outgrowth with age}

C. elegans muscle, intestine, and hypodermis exhibit a striking decline in tissue integrity as animals age (Herndon et al., 2002).
By contrast, the nervous system exhibits negligible loss of neurons or processes over adult life. Higher resolution analysis using improved imaging tools, however, reveals striking morphological changes in some aging C. elegans neurons (Fig. $1 A, B$, see example, compare young and old gentle touch receptor neurons).

To quantitatively evaluate age-associated changes in neurons, we first documented unusual morphologies evident in each of the six mechanoreceptor neurons in age-synchronized wild-type (WT) animals over adult life (visualized by cellspecific GFP expression), noting several types of novel growth from both the neurites and from the soma (Fig. 1). The gentle touch receptor neurons are situated along the cuticle, three with anteriorly positioned cell bodies (ALML, ALMR, and AVM) and three with posteriorly positioned cell bodies (PLML, PLMR and PVM) (Fig. 1 $A$, details at WormAtlas; Altun et al., 2010). ALM and PLM neurons extend a single major dendritic process anteriorly; PLM has a short dendrite that extends posteriorly from the cell body, in ALM the posterior projecting dendrite is found infrequently $(\sim 18 \%$ of ALMs in 2-d-old adults have an evident posterior dendrite in $\mathrm{p}_{m e c-4} \mathrm{GFP}$ transgenic line $z d I s 5$, see below); AVM and PVM are unipolar, with processes that first extend ventrally to enter the nerve cord and then turn to extend anteriorly for a significant length. ALMs and AVM normally form short branches close to their distal ends and these ends make synapses when they reach the nerve ring in the head. PLMs also form branches toward their distal ends with the axon branch entering the ventral nerve cord (White et al., 1986). In larvae and young adults, touch receptor processes visualized by transgenic expression of fluorescent reporters typically appear straight, with fluorescence distributed uniformly throughout the neuron (Fig. $1 A$ ).

In aging touch receptor neurons we observed specific novel morphologies at a high frequency. First, many neurons exhibit new branches that extend from the main sensory dendrite (Fig. 1C,D). The new branches appear rarely during the reproductive phase of life $(\sim 4.7$ novel branches found per 100 PLM processes at day 2 of adult life), but increase in number with age, such that by day 15 of adulthood $\left(24^{\circ} \mathrm{C}\right.$; mean lifespan is $13.1 \mathrm{~d}$ at this temperature), we find $\sim 38$ novel process branches per 100 PLMs scored (Fig. 1E). Individual processes sometimes have more than one new branch, and occasionally the novel branch can itself be branched (data not shown). Later in life, ALM and PLM processes can include numerous small extensions from their main dendrites (data not shown). Because these small extensions were barely detectable using a high-power dissecting 
microscope, we did not include them in our quantitative scoring of age-associated abnormalities (Herndon et al., 2002). Nonetheless, the presence of multiple sprout-like outgrowths in aging neurons underscores a dramatic change in structural integrity with age that features new process production.

Another prevalent type of morphological abnormality that increases in frequency with age is the extension of novel processes emanating from the soma (Fig. $1 F, G$ ). A single short process extends from the ALM soma in only $\sim 18 \%$ of young adults (Fig. $1 G)$. As is true for dendrite branches, there is an increase in numbers of neurons exhibiting one or more soma outgrowths with advancing age and this phenotype becomes prevalent later in life (Fig. $1 G$ ) (for example, $\sim 106$ novel extensions/100 ALMs scored in 15-d-old adults). Occasionally, we observed multiple outgrowths from soma dendrites, as well as outgrowths with complex branching structures on new processes that extend from the main dendrite (for example, branched branches; data not shown).

We also found that neuronal processes could adopt a wavy appearance in aging animals (Fig. $1 \mathrm{H}$, compare to straight processes in young animals in Fig. 1A). The incidence of wavy structures increased into old age (Fig. 1I). We observed wavy neurites at a frequency of $\sim 5$ per 100 PLM on day 2 , and $\sim 96$ per 100 PLM on day 10 .

We found that in some dendrites, the GFP signal appeared beaded, with concentration of fluorescence in some regions of the soma and processes but exclusion from others (Fig. $1 \mathrm{~J}$ ). Upon examination of bead-like structures at higher magnification, we noted that these beaded-looking areas often featured central dark regions that lacked GFP signal (Fig. 1J, inset). We also observed rounded structures with uniformly distributed GFP; sometimes these appeared as small balloon-like structures that appeared to extend out of the dendrite, which we refer to as protrusions (Fig. $1 \mathrm{~J}$, middle, bottom). Unlike other morphological changes, however, the incidence of beads and protrusions was highest in younger animals but declined in older PLM neurons (Fig. $1 \mathrm{~K}$ ). For example, at day 4 of adult life we find beads or protrusions in $\sim 16 / 100$ PLM processes scored; at day 10 , this measure is $\sim 3 / 100$ PLM processes.

Interestingly, in $>1100$ cell observations, we never identified neurite breaks in older animals, which would be expected if neurites become dissociated or degenerate with age. We noted similar proportions of morphological abnormalities using both GFP and mCherry expressed from the gentle touch receptor-specific mec-4
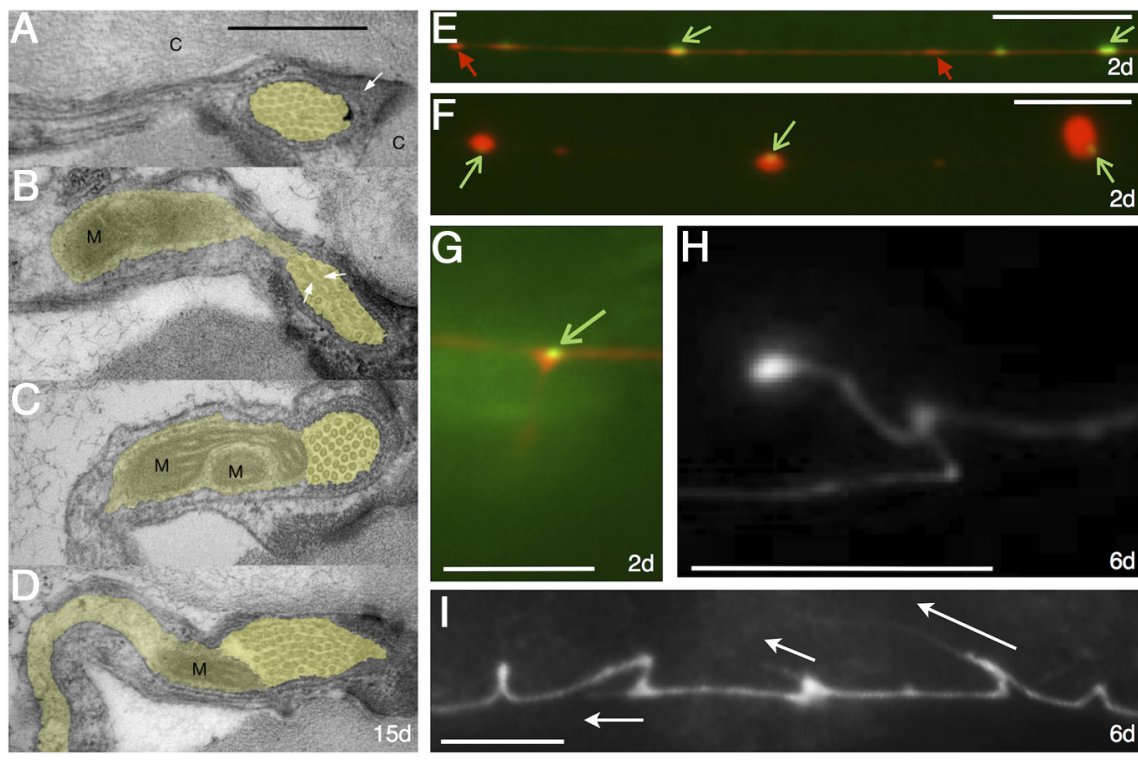

Figure 3. Aging neurons can produce multiple novel outgrowths, many of which are associated with mitochondria at the branch site. $\boldsymbol{A}-\boldsymbol{D}$, In a decrepit class ( wild-type animal at day 15 of adult life $\left(20^{\circ} \mathrm{C}\right.$ culture), the dendrite of ALMR included multiple periodic branches extending inward, away from the cuticle and the primary dendrite. Scale bar, $0.5 \mu \mathrm{m}$. $\boldsymbol{A}$, Anterior portion of the ALM dendrite (yellow overlay) shows a typical cross section view in which the narrow dendrite is virtually filled by a bundle of large-diameter microtubules (Chalfie and Thomson, 1979). The dendrite is enveloped by folding of the extremely thick body cuticle typical of old animals (Herndon et al., 2002), and the nearby extracellular space that surrounds the touch neuron is filled by a "mantle" (white arrow) and a thickened basal lamina. B, Several micrometers further posterior, the same ALM dendrite extends a narrow branch inward (also marked by yellow overlay); the branch is locally swollen to accommodate a mitochondrion (marked as M). Several microtubules in the main bundle (white arrows) seem to extend laterally toward the branch, suggesting that the branch may encompass a smaller microtubule bundle than is connected to the main bundle. $\boldsymbol{C}, \boldsymbol{D}$, Two more distinct individual branches extend inward from the same ALM dendrite, each spaced several micrometers more posteriorly from the previous branch. Inside each branch, a small mitochondrion is located close to the base of the branch. In portions of the dendrite between each branch, ALM resumes a shape and content similar to $\boldsymbol{A}$ (data not shown). Note that it is unusual to find large branches on axons in aging ALM touch neurons (Fig. 2) and the three small branches depicted in $\boldsymbol{B}$ and $\boldsymbol{D}$ probably correspond to outgrowths too small to have been scored using the dissection microscope as described for Figures 1 and 2 . Rather, these branches most likely correspond to the short tiny outgrowths we observe at $600 \times$ magnification using a compound microscope. Note also that we identified native developmental branch points in TEM micrographs in 9 young adult animals and found 8/9 for these normal branches are associated with mitochondria at the branch point, suggesting mitochondrial positioning at the branch site may be a common configuration in both normal developmental branches and aberrant novel branches that appear during aging. $\boldsymbol{E}-\mathbf{G}$, GFP-labeled mitochondria distribution in mCherry-labeled touch neuron processes with specific abnormalities. Red marker distributed throughout the cyto-

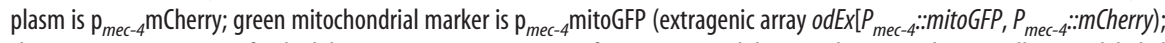
shown are PLM neurons of $2 \mathrm{~d}$ adult. $\boldsymbol{E}$, Representative process from a young adult. Note that some, but not all, green-labeled mitochondria (green arrows) are associated with beads of concentrated mCherry signal (red arrows). Scale bars: $\boldsymbol{E}-\boldsymbol{I}, 10 \mu \mathrm{m} . \boldsymbol{F}$, Representative process from a PLM neuron that features multiple beads of concentrated mCherry that appear to protrude from the main dendrite. Note that the dramatic protrusions all include an associated mitochondrion (green arrows), but some mitochondria are not associated with abnormal structures (red arrows). G, A branch site in a $2 \mathrm{~d}$ adult PLM neuron from strain with a GFP-labeled mitochondrion situated at the branch point. We find that mitochondrial GPF signals are often, but not always, associated with branch points (at day 10,90\% of new branches were associated with mitochondria, $n=40$; at day 15, 68\% of new branches were associated with mitochondria, $n=28$ ). We tracked mitochondria in PLM and find the following: (1) for the distal, native branch on which touch neuron synapses are normally made, mitochondria are present at the native synaptic branch site (combined day 2 and day 4 data $87 \%, n=39$ ), and do not decline dramatically by day 10 ( $87 \%$ of native PLM branches are associated with mitochondria, $n=32$ day 10), although there is a modest decline by day 15 (80\% native PLM branches are with mitochondria, $n=36) ;(2)$ mitochondria are dynamic and can appear and disappear within a defined region of the process in adult neurons; (3) the average number of mitochondria does not change dramatically with age (mean number of mitochondria on day 2 is 7.3 ; on day 10,7.6; on day $15,6.3 ;$ ns). FUDR was not used in quantitative studies of mitochondria summarized here. $\boldsymbol{H}, \boldsymbol{I}$, Examples of new outgrowths that appear to originate from regions of relative GFP accumulation and dendrite bending. $z d / s 5\left[p_{m e c-4} \mathrm{GFP}\right]$ was used to visualize touch neuron processes; neurons are PLMRs, $6 \mathrm{~d}$ old.

promoter (example in Fig. $1 F$ ), supporting that novel branching and outgrowth observed is not the consequence of a specific fluorescent reporter used or a specific transgene integration site. Beads and protrusions did not strongly correlate with concentration of synaptobrevin reporter SNB-1::GFP, and are unlikely to correspond to new synaptic sites on the normally synapse-free touch receptor processes (Fig. $1 \mathrm{~J}$, legend). 
A

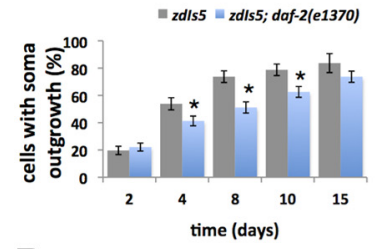

D

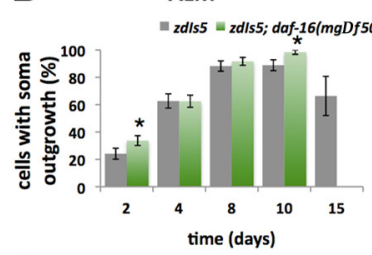

G

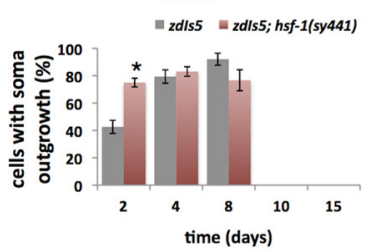

$J=h s f-1($ sy441) $=h s f-1($ sy441); $h s f-1+$

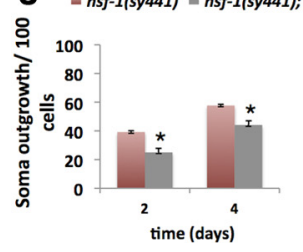

B

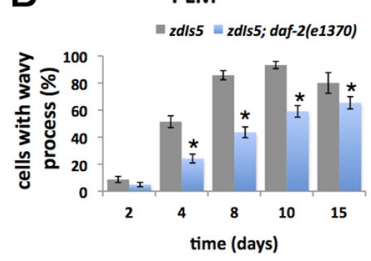

$\mathrm{E}$

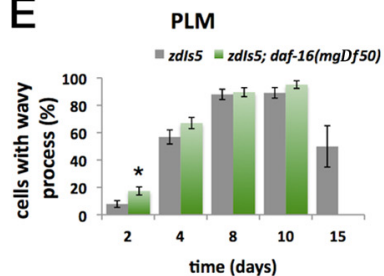

$\mathrm{H}$

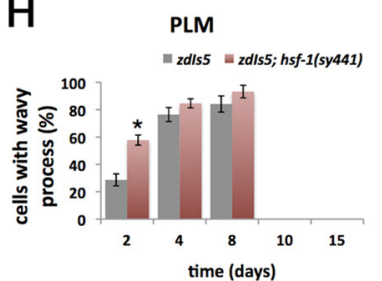

$\mathrm{K}$

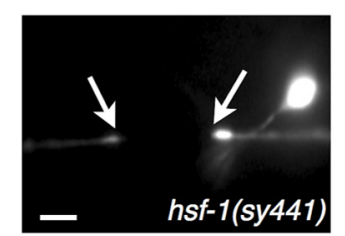

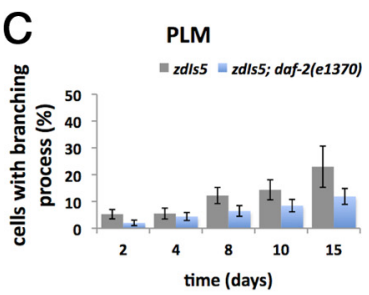
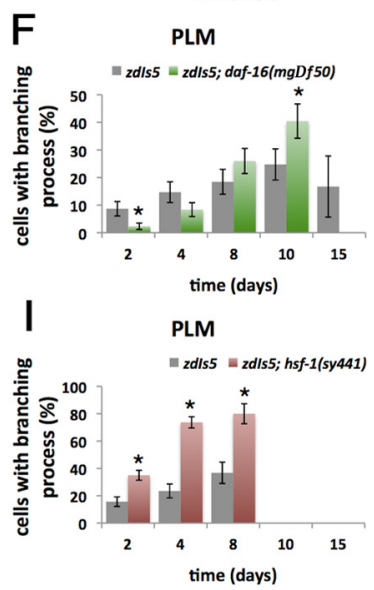

$\mathrm{L}$

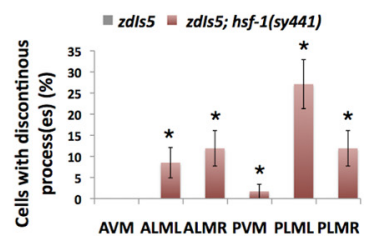

Figure 4. Insulin signaling affects age-associated morphological changes in adult touch neurons. $\boldsymbol{A}-\boldsymbol{K}$, Wild-type, reductionof-function insulin receptor daf-2(e1370), transcription factor FOXO/daf-16(mgDf50) null mutant, and temperature-sensitive

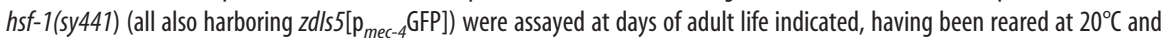
shifted to $24^{\circ} \mathrm{C}$ at the $L 4$ larval stage. Numbers of neurons scored for common phenotypes of ALM soma outgrowth $(A, D, G), P L M$ wavy appearance $(\boldsymbol{B}, \boldsymbol{E}, \boldsymbol{H})$, and PLM novel process branching $(\boldsymbol{C}, \boldsymbol{F}, \boldsymbol{I})$ were as follows: $z d l s 5$ (data for wild-type control assayed in daf-2 experiments): 172 day 2, 128 day 4, 106 day 8,90 day 10,30 day $15 ; z d / 55$; daf-2: 198 day 2,182 day 4, 156 day 8,144 day 10 , 118 day 15; zdls5 (wild-type control assayed in daf-16 experiments): 116 day 2, 88 day 4, 76 day 8, 64 day 10, 12 day 15; zdls5;daf-16(mgDf50): 172 day 2, 130 day 4, 96 day 8, 62 day 10 (by day 15 all daf- 16 animals were dead); $z d l s 5$ (wild-type control assayed in $h s f-1$ experiments): 54 day 2, 34 day 4, 19 day 8; zdls5; hsf-1(sy441): 90 day 2, 59 day 4, 15 day 8 (no live hsf- 7 mutants on adult day 10). Asterisk for $p<0.05$ in mutant versus WT control on the indicated day, significance was determined using Fisher's exact test. We observed similar outcomes for studies of aging touch neurons in daf-2(e1368); our daf-2 and daf-16 data are consistent with findings in Pan et al. (2011) and Tank et al. (2011) (daf-16(mu86)), although those studies did not feature a focus on specific abnormalities and individual touch neuron types. $J$, Cell autonomous expression of $h s f-1$ in touch neurons rescues early onset elevation of the ALM soma outgrowth phenotype. We expressed $h s f-1$ specifically in the touch neurons under the control of the mec- 4 promoter, in the background of $h s f-1(t s) 20^{\circ} \mathrm{C}$ (see Materials and Methods) and compared novel soma outgrowth to control animals that expressed only the $\mathrm{p}_{\text {mec }-4} \mathrm{mCherry}$ construct used to visualize neuronal processes. Interestingly, expression of hsf- 1 in the PLM neurons was associated with the presence of blunted dendrites in $\sim 50 \%$ of neurons observed (data not shown), suggesting a dominant-negative effect on process maintenance (and possibly elevated breaking) for this subset of neurons; AVM and PVM are not significantly different from non-rescued lines. $\boldsymbol{K}$, Example of a 4-d-old hsf-1 mutant touch neuron process (horizontal fluorescence) with an apparent break, ends indicated by arrows. Another touch neuron soma can be seen in the middle-right background. Similar beading at broken ends is seen immediately after laser axotomy. Scale bar, $10 \mu \mathrm{m}$. L, Cellspecific quantitation of touch neuron "breaks" in the hsf-1(sy411) background on adult day 4. Strain measured is zdls5; hsf1(sy441): 90 day 2, 59 day 4,15 day 8.

We conclude that aging touch receptor neurons exhibit morphological changes, most often in the form of novel branches from processes or somata and/or by adopting wavy appearances, but rarely, if ever, by extensive axonal degeneration. Similar observations of age-associated novel outgrowths in touch neurons have been recently reported (Pan et al., 2011; Tank et al., 2011). The fact that the same neurons may, or may not, develop structural abnormalities in different animals may reflect stochastic

molecular crises that can influence aging (Herndon, 2002; Rea et al., 2005) or microenvironment differences.

\section{Different classes of touch receptor neurons exhibit differences in age-associated morphological changes}

We combined scores for novel process outgrowth, soma outgrowth and process waviness to evaluate the numbers of each touch receptor neuron that exhibited any morphological abnormality at a given time in adult life (Fig. 2A). Overall, it is clear that neuronal abnormalities increase in frequency as animals age, yet some classes of touch neurons showed more dramatic abnormalities than others.

To look more closely at the heterogeneity of aging phenotypes in individual touch receptors, we evaluated the relative frequencies of specific types of morphological changes for each neuron over time. Figure $2 \mathrm{~B}$ compares process branching, soma outgrowth, and waviness frequencies among individual touch neurons at day 10 of adult life, $24^{\circ} \mathrm{C}$. Although the touch receptor neurons share developmental programs, morphological features, and functional similarities (Goodman, 2006), we found considerable differences in the abnormality type exhibited by touch neuron subtypes.

ALMs express a high degree of new somata outgrowth $(\sim 79 \%$ of ALMs scored at day 10), but virtually no somata outgrowth was observed for PLMs at $10 \mathrm{~d}$. In contrast, PLMs exhibit substantial new branch outgrowth from the main process ( $\sim 20 \%$ of PLMs scored), with virtually no process branches for ALMs at this time point. PLM neurons also show a much higher frequency of wavy processes at day 10 than do ALMs (>90\% PLMs are wavy at this time point as compared with $13 \%$ of ALMs). Biases for one abnormality type over another in a specific neuron subclass persisted over adult life (data not shown). We did not find any statistically significant differences between left/ right pairs ALML/ALMR or PLML/ PLMR. Although AVM and PVM do not exhibit as much morphological change as the ALMs and PLMs, both soma outgrowth and wavy appearance increase with age in AVM/PVM, with times of onset and frequencies that differ between these two neurons (data not shown).

We conclude that heterogeneity of age-associated changes can transpire within one specific neuronal type and infer that touch receptor neurons are differentially susceptible/responsive to ageassociated factors that promote novel outgrowth from soma, branches from processes, or wavy trajectories of dendrites. 
Whether differences arise from body position, local environment, extent of mechanosensory input during life history, unique neuron-specific molecular features, or a combination of these factors is unknown.

\section{Novel branches in native aging touch receptor neurons are often associated with mitochondria at the branch point}

We noted a high frequency of novel growth abnormalities in aging touch receptor neurons as visualized by multiple fluorescent reporters (Figs. 1,2). To determine the basic cell biological features of these new outgrowths and rule out that they might be artifacts of expressed transgenes, we sought to find them in electron micrographs from native aging neurons. We reexamined EM thin sections of aging touch receptor neurons that were originally studied in wild-type non-transgenic animals that had aged to a decrepit state of highly diminished locomotory function, but were not preselected for neuronal abnormalities (Herndon et al., 2002). Indeed, we were able to identify multiple novel outgrowths in a touch neuron dendrite in the head of a serially sectioned individual by systematic searching. Figure $3 A-D$ shows representative serial sections from different regions of an ALM of a decrepit animal in its 15th day of adult life $\left(20^{\circ} \mathrm{C}\right)$, which exhibited considerable locomotory impairment (Herndon et al., 2002). Analysis revealed the existence of three distinct small processes extending from the main dendrite, each one characterized by the presence of a microtubule network (MT), and the positioning of a mitochondrion at the base of the branch. Note that the small outgrowths we show in Figure $3 B-D$ most likely correspond to tiny extensions in old touch neurons that we do not score as major abnormalities.

We further examined mitochondria distribution relative to morphological abnormalities in adult touch neurons using a GFP-tagged mitochondrial matrix protein in a line that also expresses mCherry in touch receptor cytoplasm (Fig. 3E,F). We find some, but not all, beadings on processes correlate with mitochondria, both for bulges that appear within processes (Fig. 3E; example of these structures in Fig. $1 \mathrm{~J}$, top) and those large varicosities that appear to extend off from processes (Fig. 3F; example of this structure in Fig. $1 \mathrm{~J}$, middle panel). As expected from our EM data, we also often find mitochondria at branch sites in aging neurons (data in legend of Fig. $3 G$; mitochondria are also associated with the native developmental touch neuron branch). Touch neuron mitochondria do not change much in number as animals age (data in legend of Fig. $3 G$ ). Whether mitochondria might themselves induce branching or might be recruited to, or trapped at, sites of damage (e.g., microtubule breaks or protein aggregates) remains to be determined. It does appear that novel outgrowths can emerge from sites of obvious axon bending, which have to involve discontinuities in the MT network (Fig. $3 H, I)$.

Our EM data establish that novel outgrowth occurs during aging of native C. elegans neurons, and therefore cannot be attributed to introduced transgenes. Moreover, EM data suggest that the production of small branches is common in aging neurons-even neurons such as ALM that do not typically express major new branches readily discerned using a dissecting microscope (Fig. 2) can be easily found to have multiple small projections. Finally, although numbers of mitochondria in the neuronal process do not diminish dramatically with age, mitochondria positioning at the base of the new process outgrowth appears to be a relatively common feature of adult morphological abnormalities, raising questions about the role of mitochondria in the age-associated structural changes that characterize touch receptor neurons.

\section{The insulin signaling pathway influences morphological changes in aging touch neurons}

The insulin/IGF pathway influences longevity across phyla (Suh et al., 2008; Broughton and Partridge, 2009) and low insulin signaling has also been shown to be associated with extended healthspan (Garigan et al., 2002; Herndon et al., 2002; Gerstbrein et al., 2005). Low insulin signaling can be neuroprotective in $C$. elegans and in mammalian models of age-associated insults such as protein aggregation (Cohen and Dillin, 2008; Cohen et al., 2010). To determine the impact of the insulin/IGF signaling pathway on morphological aging of neurons, we first constructed $\mathrm{p}_{\text {mec-4 }} \mathrm{GFP}$ lines that had longevity-extending reduction-offunction mutations in InsR daf-2. We tracked age-associated morphological changes in individual WT and mutant touch receptor neurons over adult life.

We initially focused on the effects of reducing insulin signaling on the most common age-associated touch neuron morphological changes that we documented in Figures 1 and 2. We found that ALM soma outgrowths in daf-2(rf) mutant e1370 are reduced later in adult life, with significant differences first detected at day 4 of adult life as compared with wild-type animals (Fig. $4 \mathrm{~A}$, similar data for daf-2(e1368), data not shown). Likewise, the frequency of wavy-appearing processes in PLM neurons was diminished by daf-2(rf), with differences first becoming apparent at day 4 and persisting late into adult life (Fig. $4 B$ ). There is a trend toward neuroprotection for the novel axon branch phenotype common in PLM neurons, although differences for this particular morphological change do not reach statistical significance (Fig. $4 C ; 0.05<p<0.2$, Fisher's exact text). Interestingly, we also noted a significantly elevated frequency of middle-age and old ALM and PLM neurons expressing the young adult "beaded" phenotype throughout adult life in daf-2(rf) (data not shown). These data complement other studies (Pan et al., 2011; Tank et al., 2011) in revealing a role for DAF-2 InsR and insulin signaling in morphological aging of $C$. elegans touch receptor neurons.

\section{DAF-16/FOXO plays a role in neuroprotection}

We next examined morphological profiles of aging touch neurons in a daf-16 null mutant that mimics high insulin pathway activation, shortens lifespan, and accelerates aging (Ogg and $\mathrm{Ru}-$ vkun, 1998; Liu et al., 1999). We found that in 2-d-old young adults, the incidence of ALMs with soma outgrowth (Fig. 4D) and PLMs with wavy appearance (Fig. $4 E$ ) was increased, consistent with a role of DAF-16 in neuroprotection and a progeric effect for its deletion. However, the effect of daf-16( $\Delta)$ is strikingly limited: (1) the magnitude of increase of early defects at day 2 in daf-16 mutants is relatively modest, and (2) there are no differences between WT and daf-16 for ALM soma outgrowth on days 4 and 8 or PLM waviness on days 4,8 , and 10 , and differences in PLM branching are only apparent at day 10 . Our data suggest that in addition to some daf-16-dependent influences, daf-16independent mechanisms are likely to exert a significant influence on morphological neuronal aging.

We also note that we observed a few modest, but statistically significant, neuronal outgrowth responses to insulin-like signaling (IIS) pathway manipulation that suggest a complex equation between morphological aging, cell type, and insulin signaling. For example, we observed an age-associated increase in the rare phenotype of ALM process branching in the daf-2 background (for example, day 10 control 1\%; daf-2 $2 \%$ ); PVM (but not AVM) exhibits an increase in process branching in the daf-2 background (for example, day 10 control $0 \%$; daf-2 $\sim 6.9 \%$ ), and for the more prevalent age-associated PLM process branching in 
the daf-16 mutant, PLM branching is initially decreased (day 2) and later increased (by day 10) relative to wild-type (Fig. 4F). Together, these observations suggest that distinct morphological changes in different neurons might be differentially impacted by the IIS pathway.

Heat shock factor-1 transcription factor is neuroprotective against early onset morphological abnormalities and dendrite breaks

In addition to repressing DAF-16 activity, insulin signaling also promotes aging by repressing heat shock factor- 1 transcription factor $(h s f-1)$, which can function with DAF-16 to promote proteostasis when active (Garigan et al., 2002; Hsu et al., 2003; Morley and Morimoto, 2004; Cohen et al., 2006). Given that daf-16( $\Delta)$ exhibited relatively little influence on morphological aging of neurons, we tested whether HSF-1 might modulate morphological neuronal aging phenotypes. In these studies we used the temperature-sensitive $h s f-1$ (sy441) strain, switching to restrictive temperature at the late L4 stage. We observed striking early onset increases in morphological outgrowth in aging touch neurons when HSF-1 activity was reduced (Fig. 4G-I). More specifically, common morphological abnormalities of ALM soma outgrowth, PLM waviness, and PLM branching were all increased at day 2 of adult life, evidence of a significant progeric effect of $h s f-1$ inactivation. Aberrant outgrowth was also increased in AVM and PVM neurons (data not shown).

To ask whether $h s f-1$ acts cell autonomously to influence ageassociated morphological changes, we expressed $h s f-1$ only in touch neurons in the $h s f-1$ mutant background (Fig. $4 J)$. We found that early onset soma outgrowth in $h s f-1$ mutant ALMs could be rescued by touch-neuron specific expression, indicating that $h s f-1$ acts within the ALM neurons to maintain neuronal architecture in early adult life.

The $h s f-1$ mutant also exhibited an abnormality not seen in aging wild-type neurons-processes frequently appeared to feature full breaks (Fig. $4 K, L$ ). Breaks were apparent in the aging $h s f-1$ mutant at fairly high frequency (for example, at day 4: 27\% PLML, 12\% PLMR, 9\% ALML, and 12\% ALMR appeared broken). Thus, HSF-1 may directly maintain or repair process integrity in adult life or may act via other tissues to exert systemic positive effects on nervous system maintenance. Interestingly, in the $\operatorname{Ex}\left[p_{\text {mec- } 4} h s f-1\right] ; h s f-1$ line, $\sim 50 \%$ of the PLM neurons have dendrites that appear truncated (Fig. $4 \mathrm{~J}$, legend), suggesting a cell-autonomous dominant-negative effect of elevated expression of $h s f-1$ on these neurons. The distinct "rescue" phenotypes in ALM versus PLM again indicate differential susceptibility/responses of aging neurons, even for highly similar neuronal types.

We conclude that HSF-1 contributes a major activity that normally protects against age-associated process outgrowth and that protects against break persistence. Since $h s f-1$ can act downstream of insulin signaling, reduced insulin signaling might potentiate this effect (although $h s f-1$ can be activated via other pathways as well). A general decline in protein folding efficiency, maintained biosynthesis of seemingly irrelevant proteins late in life [for example, production of yolk protein after oocytes are depleted (Herndon et al., 2002)], and relaxation of miRNA-mediated translational repression (IbanezVentoso et al., 2006), may all add to protein proteostasis challenges that accompany aging. Given that HSF-1 plays a major role in chaperone expression and maintenance of proteostasis (Fujimoto and Nakai, 2010) and has been specifically shown to
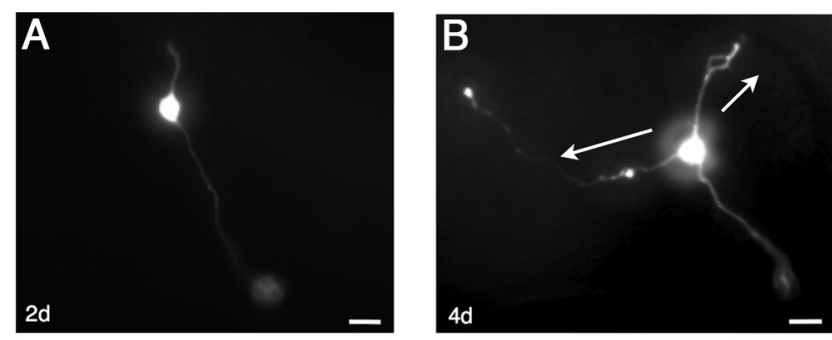

C

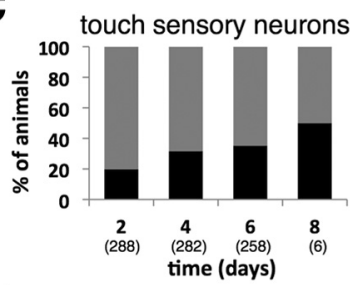

E

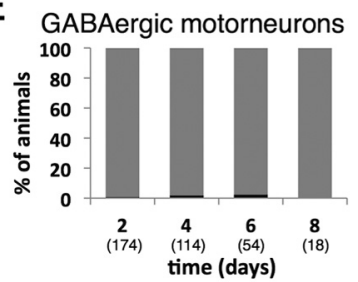

cells with abnormalities
D dopaminergic PDE neurons

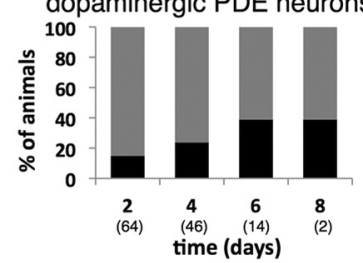

$\mathbf{F}$

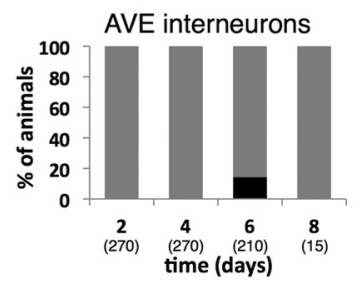

unaltered cells

Figure 5. Age-associated morphological changes are exhibited by some, but not all, $C$. elegans neurons. $\boldsymbol{A}, \boldsymbol{B}$, Dopaminergic PDE sensory neurons exhibit novel branching phenotypes with increasing age. Shown are as follows: $\boldsymbol{A}$, young PDE; $\boldsymbol{B}, 4$-d-old PDE exhibiting a novel branch structure; transgene array is egls $7\left[p_{\text {dat- } 7} \mathrm{GFP}\right]$. Scale bars: $10 \mu \mathrm{m} . \boldsymbol{C}-\boldsymbol{F}$, Incidence of detectable abnormalities on representative neuronal types. $\boldsymbol{C}$, Mechanosensory touch receptor neurons; transgene array zdls5[p$\left.p_{\text {mec-4 }} \mathrm{GFP}\right] ; \boldsymbol{D}$, dopaminergic $\mathrm{PDE}$; transgene array egls1 $\left[p_{\text {dat }-1} \mathrm{GFP}\right] ; \boldsymbol{E}, \mathrm{GABAergicmotorneuron} \mathrm{commissures;} \mathrm{transgene} \mathrm{array} \mathrm{ox/s} 12\left[p_{\text {unc- } 47} \mathrm{GFP}\right] ; \boldsymbol{F}, \mathrm{AVE}$ glutamatergic interneurons; transgene array gvEx173[ $\left.\left.p_{\text {opt-3 }} \mathrm{GFP}\right]\right) . x$-axis is days of adult life, numbers of cells examined are given in parentheses; red bars indicate percentage cells with abnormalities. Differences in successive trial samples were significant by $t$ test comparison $p<$ $0.001[C: 2,4 ; 4,6 ; 6,8 ; \boldsymbol{D}: 2,4 ; 4,6 ; 6,8, \mathrm{~ns} ; \boldsymbol{E}$ : values $(0.7,1.8,2.3,0)$ are significantly different but not clearly shown here because we elected to keep the same scale or each graph to emphasize differences among neuronal types]. Note that in this study, we followed the same neurons in the same animals during adult life, scoring the animals on successive days. This longitudinal survey with repeated mounting and high light intensity irradiation limited viability of the strains we examined, and thus we were unable to record data for very old animals, which all exhibited significantly reduced survival (data not shown).

promote protein disaggregation activity in C. elegans (Cohen et al., 2006), protein aggregation might be a significant factor in promoting novel outgrowth, possibly even inducing transient breaks, in aging neurons.

Age-associated morphological changes are exhibited by some, but not all, $C$. elegans neurons

We wondered whether morphological changes similar to those we observed in touch receptor neurons are prevalent throughout the aging $C$. elegans nervous system. To begin to address this question, we examined representative neuronal types, focusing on fluorescent reporters that visualize small subsets of neurons (or regions of neurons) to facilitate high-resolution analysis of individual processes (Fig. 5).

In the C. elegans hermaphrodite, eight neurons produce dopamine-two pairs of CEP neurons with soma positioned midpharynx and sensory processes extending anteriorly to the tip of the nose, a pair of ciliated ADEs that send processes anteriorly from cell bodies positioned near the end of pharynx to the central 
isthmus region of the pharynx, and a pair of PDE neurons with somata positioned laterally in the mid posterior and processes that extend down to the ventral nerve cord to bifurcate and run anteriorly to the pharynx and posteriorly to the anus (Sulston et al., 1975). These dopaminergic neurons play a role in mechanosensation of bacterial food (Sawin et al., 2000) and can be visualized by expression of a $\mathrm{p}_{\text {dat }-1} \mathrm{GFP}$ reporter. We examined the PDE process, with a focus on its primary extension into the ventral nerve cord (Fig. $5 A, B$ ). As we found for the gentle touch receptor neurons (Fig. 5C), PDE neurons exhibit aberrant morphologies that increase in frequency with age, such that by day 6 of adult life $\sim 40 \%$ of PDEs examined exhibited novel growths (Fig. 5D). We did not observe major branching in other dopaminergic neurons. Thus, at least two distinct mechanosensory neuron types, touch receptors and PDEs, exhibit increased morphological abnormalities with age.

Not all neurons we examined, however, exhibited extensive age-associated morphological changes. Inhibitory GABAergic DA and DB motoneurons, with somata positioned along the ventral cord, send commissures from ventral to dorsal nerve cord that can be individually observed using a $\mathrm{p}_{\text {unc-47 }} \mathrm{GFP}$ reporter (McIntire et al., 1997). We examined commissures in aging adults, but only observed occasional novel branch abnormalities (Fig. 5E). Two nerve ring interneurons (AVEL and AVER), which have somata in the lateral ganglia next to the nerve ring and extend single processes posteriorly to the mid-body, express reporter $\mathrm{p}_{\text {opt-3 }} \mathrm{GFP}$ (Fei et al., 2000). We examined these neurons over adult life, but did not observe novel axon branches or process extensions from the soma (Fig. $5 F$ ).

We conclude that not all neurons exhibit new outgrowth morphologies in the aging C. elegans nervous system. Our data reveal heterogeneity in susceptibility to age-associated changes according to cell type.

\section{Novel outgrowth phenotypes do not eliminate touch sensitivity}

Mutations in C. elegans nucleotide-gated sensory channel subunits tax-2 and tax-4 lead to abnormal axon branching in adults (Coburn et al., 1998). Calcium channel mutations or disruption of sensory cilia have also been associated with aberrant branching (Peckol et al., 1999). Thus, neuronal inactivity might be suggested to induce neuronal outgrowth. To get a first indication of the relationship of touch sensitive function and morphological abnormality in the six touch receptor neurons, we probed for statistical association of novel outgrowth and touch defects (data not shown).

We scored $\mathrm{p}_{\text {mec- } 4} \mathrm{GFP}$ animals for anterior and/or posterior sensitivity to record touch response phenotypes to 5 successive touches. A positive response was scored for even modest cringing in response to touch, because bodywall muscle deterioration, which impairs locomotion, is striking by late time points (Herndon et al., 2002). We then scored touch receptor neurons for abnormalities. We looked for associations between morphological abnormalities and touch sensitivity in two ways. First, we identified all animals scored as having anterior touch defects and we asked whether these animals differentially had abnormalities in all three anterior touch neurons (ALMs and AVM); likewise, we identified all animals scored to have behavioral posterior touch insensitivity and asked whether these differentially had morphological abnormalities in the posterior gentle touch PLM neurons [PVM does not mediate a gentle touch response (Chalfie et al., 1985)]. Second, we compared occurrence of morphological abnormalities between animals having anterior/posterior touch defects and animals with intact touch sensation.
We find no statistically significant correlation between morphological abnormality and defective touch response. Thus, the presence of morphological change in a touch receptor neuron does not indicate a complete loss of function of the affected neuron. However, we emphasize that this analysis cannot address whether a partially diminished activity is associated with morphological aberration in aging touch neurons (Pan et al., 2011; Tank et al., 2011).

\section{Synaptic integrity diminishes with age, with extent of deterioration correlated with locomotory capacity}

In aging human brain, synaptic efficacy rather than cell loss is thought to underlie functional decline (Yankner et al., 2008; Bishop et al., 2010; Fjell and Walhovd, 2010). To get a higher resolution view of the aging $C$. elegans nervous system and to address whether synaptic deterioration is a prominent aspect of age-associated decline in this model, we compared ultrastructural features of synaptic integrity in 1-2-d-old young adults and 15-d-old aging adults selected from locomotory Class A (maintained locomotory vigor; graceful agers) and Class C (decrepit, nearly paralyzed animals; poor agers) [features of locomotory classes discussed by Herndon et al. (2002)]. In these studies, we focused on electron micrographs of the nerve ring and ventral ganglion, where the majority of synapses are made (White et al., 1986).

As in higher organisms, C. elegans chemical synapses are defined by the presence of a presynaptic dense bar and the presence of synaptic vesicles nearby (corresponding to the active zone of vesicle release; evident as vesicle-rich synapses in Fig. $6 \mathrm{~A}$ ). There is no visible postsynaptic specialization in nematode synapses. In rare instances in young adult animals, we noted a depleted chemical synapse in the nerve ring, in which there were few vesicles lying close to the presynaptic bar (Fig. 6B). Presynaptic densities could still be recognized in old animals, enabling us to count vesicles in the vicinity of the density (Fig. $6 C$ ). We found that vesicle-depleted synapses were more common in $15 \mathrm{~d}$ Class A animals (Fig. 6D,E), and were the predominant form in Class $\mathrm{C}$ animals (Fig. 6E). Similarly, the average number of vesicles per synapse was significantly lower in $15 \mathrm{~d}$ Class A animals than in young adults, and extremely low in $15 \mathrm{~d}$ Class $\mathrm{C}$ animals. In depleted synapses, the overall size of the presynaptic terminal was generally reduced in cross-section, in proportion to the change in gross vesicle numbers, with the terminal diameter scaling in direct proportion to the square root of the number of vesicles within the presynaptic swellings (Fig. 6E, see Materials and Methods). Importantly, the differences in synaptic structure between same aged-old animals that have aged well (maintained synaptic structure) and have aged badly (more deteriorated synaptic structure) suggest that nervous system integrity may be a valid reporter of healthspan.

In addition to significant structural changes at the synapse, processes and neuronal soma in aged animals can appear different from those in younger neurons. Age-associated changes include axon thinning, occasional accumulation of vesicles in processes suggestive of failed axonal transport, increased electron dense accumulation near the synapse but decreased electron density in axonal cytoplasm suggestive of maintained microtubule network but depletion of other organelles, and shrinking of soma and nuclear volume despite maintained chromatin demarcation (data not shown).

We conclude that: (1) synaptic integrity markedly declines in aging $C$. elegans with a significant reduction in synaptic vesicle content as a predominant feature; and (2) old animals that have 

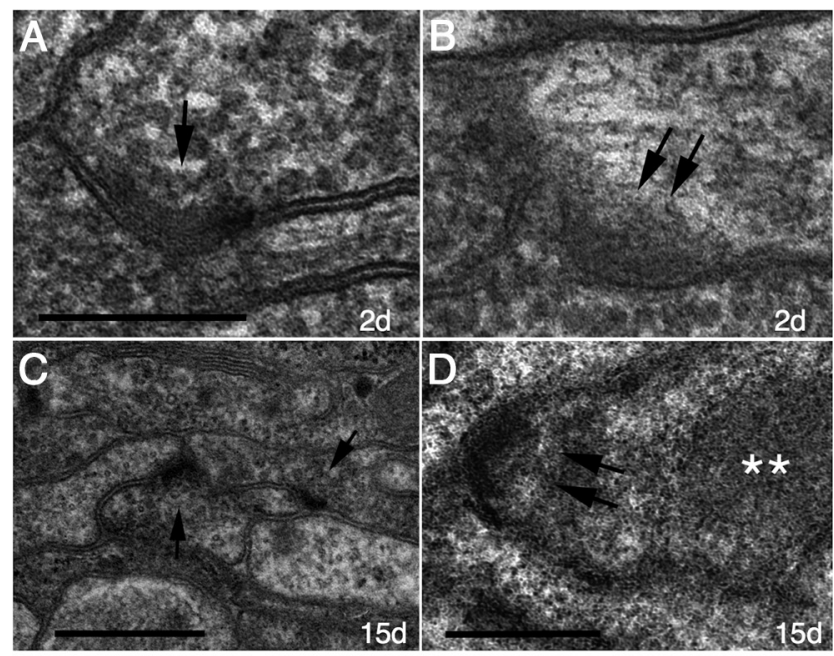

E
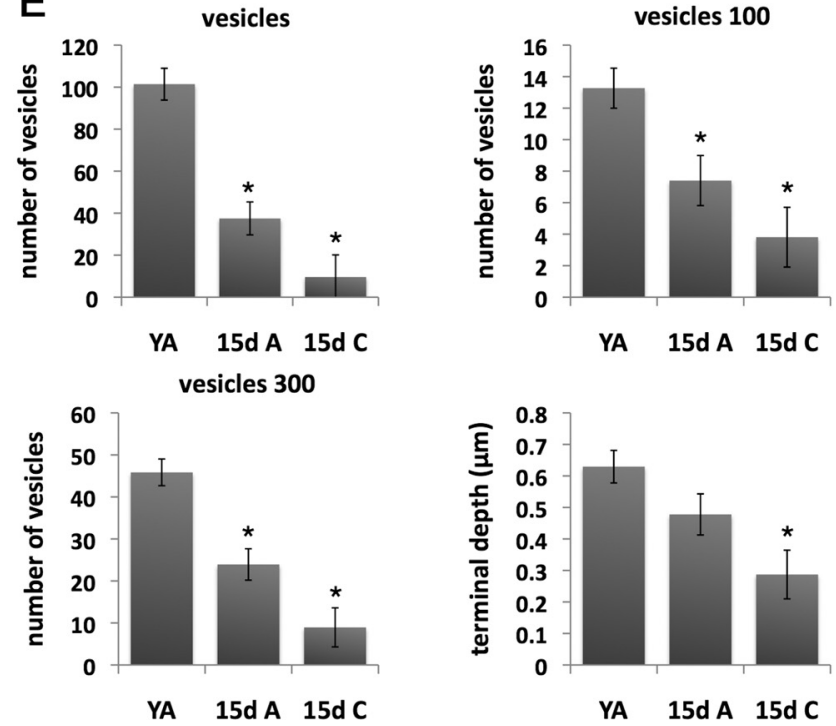

Figure 6. C. elegans synapses deteriorate with age, with extent of synaptic reduction correlated with degree of mobility impairment. $\boldsymbol{A}$, Closeup of a normal synapse (arrow) in a young adult animal. A prominent presynaptic bar lies along the plasma membrane, and the process is swollen with synaptic vesicles. Vesicles lying close to the bar are somewhat smaller in diameter than vesicles away from the release zone. There is no anatomical specialization seen on the postsynaptic process. Scale bars: $\boldsymbol{A}, \boldsymbol{B}, 0.25 \mu \mathrm{m}$. $\boldsymbol{B}$, A depleted synapse (double arrows) in the same young adult displays a normal presynaptic bar, but a paucity of synaptic vesicles close to the bar or at a distance. $C$, In a Class $A$ adult at $15 \mathrm{~d}$ of adulthood $\left(20^{\circ} \mathrm{C}\right)$, chemical synapses (arrows) remain well organized but have fewer vesicles near the presynaptic bar and the presynaptic process is therefore smaller in diameter. Note that many nearby axons (away from the synapse) remain almost the same diameter as in a young adult. Many axons still contain clusters of synaptic vesicles and small bundles of microtubules. Scale bar, $0.5 \mu \mathrm{m}$. D, Closeup of a depleted synapse (double arrows) in a Class C animal at $15 \mathrm{~d}$ of adult life. A fuzzy electron dense inclusion (white asterisks) lies close to the depleted synapse. This may represent pathological deposition of cytoplasmic proteins. Scale bar, $0.25 \mu \mathrm{m}$. $\boldsymbol{E}$, Quantitation of synaptic features in aging $C$. elegans. YA, Young adult; $15 \mathrm{~d} A, 15$-d-old class $A$ animal $\left(20^{\circ} \mathrm{C}\right)$ that is relatively vigorous for its same-age counterparts and considered to have aged gracefully; $15 \mathrm{dC}, 15$-d-old class C animal that is decrepit, barely mobile, and considered to have aged poorly. Data include measurements of 51 synapses from 6 young adults; 52 synapses from 3 Class $A$ animals; 28 synapses from 3 Class C animals. Synapses were from the nerve ring and lateral ganglia. "Vesicles" indicates counts of all vesicles; "vesicles 100 " counts all within $100 \mathrm{~nm}$ of the synaptic density; "vesicles 300" scores all within $300 \mathrm{~nm}$ from the synaptic density. Asterisks indicate $p<0.02$ as compared with young adult values; repeated-measures ANOVA test (SAS program). For terminal length, YA compared with $15 \mathrm{~d} A, p=0.10$. See notes on measuring terminal length in Materials and Methods. aged poorly (Class C, as judged by locomotory criteria) have significantly diminished synaptic structure as compared with their same-age siblings that have aged well (Class A, as judged by locomotory criteria). We find that the decline of synapses and other neuronal structures parallels locomotory impairment and thus synaptic deterioration might contribute to the progressive mobility dysfunction that occurs during aging. Other muscle function loss, such as accompanies pharyngeal pumping decline (Huang et al., 2004) might also have a synaptic component. Importantly, the synaptic decline observed in aging C. elegans is strikingly reminiscent of that proposed to contribute to human brain aging, raising the possibility that common mechanisms of decline might be operative.

\section{Discussion}

We report that aging C. elegans neurons can exhibit novel neurite outgrowth from dendrites and from somata. New outgrowths can be highly prevalent in aging touch receptor neurons, with mitochondria often positioned at branch sites. Different neurons exhibit distinct types of outgrowth, even with a single neuronal class. However, not all neurons exhibit morphological change with age, demonstrating cell-specificity of structural decline. Insulin signaling modulates adult architectural maintenance. Synaptic deterioration is also prevalent in the aging $C$. elegans nervous system, featuring diminished presynaptic density size and lower synaptic vesicle numbers as animals get older. Commonalities with aging mammalian brain suggest conserved mechanisms may be operative in neuronal decline across phyla.

\section{Different neurons exhibit different susceptibility to age- associated morphological change}

Many genes implicated in inherited human late onset neurodegenerative diseases are expressed broadly yet are preferentially deleterious only in one cell type. The nature of differential susceptibility of specific neuronal types to particular stresses is not well understood, although it is clear that age is a major contributing factor. We document that structural aging of the C. elegans nervous system exhibits a striking differential susceptibility, with some neurons exhibiting virtually no dendritic restructuring, whereas in some others, virtually all aged neurons exhibit some regrowth abnormality (Fig. 5). Major differences can even occur among neurons of one specific type-for example, differences in the morphological abnormality type of anterior versus posterior touch neurons (ALMs, high frequency soma outgrowth; PLMs, elevated dendrite branching; Figs. 1,2), as well as differences in the response to molecular manipulation ( $h s f-1$ expression in $h s f-1$ mutant ALMs rescues accelerated outgrowth phenotypes; expression in PLMs causes truncated processes, Fig. $4 J$ ). Differences in susceptibility to resprouting in old age might be affected by neuronal position (neuronal anchoring, quality of extracellular matrix, available "room to grow"), cell-specific gene expression (e.g., genes that encode oxidative stress defenses, protect against mechanical disruption, promote proteostasis and effective axon transport, and/or maintain synaptic integrity). The $C$. elegans model is particularly well suited for testing such hypotheses via single neuron gene profiling, overexpression and/or knockdown of candidate genes, genetic manipulation of neuron position and fate, and unbiased genetic screens for factors that alter neuronal susceptibility. Future characterization of the factors influencing the aging of specific neurons in the $C$. elegans model might provide insight into differential susceptibility in late-onset disease. 


\section{Could mitochondrial positioning at age-associated branch} sites drive new dendrite formation?

Mitochondrial dysfunction and genome mutations have been implicated in age-associated decline across species (Martin, 2010). Mitochondrial number in touch neuron dendrites does not decline significantly with age (Fig. $3 G$, legend). Somewhat unexpectedly, we find touch neuron mitochondria often positioned at the branch point for major (Fig. 3G) and tiny (Fig. $3 B-D)$ age-associated outgrowths. Mechanisms that might lead to positioning of mitochondria in branches induced in aging neurons include the following: (1) mitochondria might be normally needed to support energy requirements for new outgrowth; (2) mitochondrial transport might become blocked by trafficking dysfunction/protein aggregation, causing a physical obstruction that promotes cytoskeletal assembly in a new direction; or (3) dysfunctional mitochondria might cause local energy charge deficiencies or aberrant ROS signals that induce branching. Indeed, mitochondrial mutant mev-1, characterized by increased oxidative stress, can exacerbate branching, although not all oxidative stresses do so (Tank et al., 2011).

\section{Longevity-promoting insulin signaling influences morphological aging of touch neurons}

Reduced insulin/IGF signaling is a conserved longevity mechanism (Broughton and Partridge, 2009), and IIS within the nervous system itself appears of high importance for longevity benefits, both in C. elegans (Wolkow et al., 2000; Iser et al., 2007) and mouse (Taguchi et al., 2007). We show that reduced insulin signaling $($ daf-2(rf $))$ positively influences the morphological aging of $C$. elegans neurons (Fig. 4A-C), and conversely, deletion of the DAF-16/FOXO transcription factor, a genetic condition that mimics high insulin pathway signaling, induces progeric morphological changes for some neurons (Fig. 4D-F). Our data extend and confirm recent studies (Pan et al., 2011; Tank et al., 2011) to show that age-associated morphological phenotypes in C. elegans neurons can be positively modulated by lowering insulin/IGF-like signaling. Interestingly, insulin/IGF signaling can influence dendritic branching in vertebrate neurons (Chiu and Cline, 2010), raising the possibility that insulin-dependent morphological responses might reflect a common function across species.

\section{HSF-1 protects against age-associated morphological changes} in touch receptor neurons

Our data also identify the transcription factor $h s f-1$, which can act downstream of DAF-2 (Hsu et al., 2003; Morley and Morimoto, 2004), as a significant regulator of neuronal morphology. At nonpermissive temperature, $h s f-1$ mutant touch neurons exhibit early onset increase in all age-associated changes we monitoredsoma outgrowth, dendrite branching, and wavy conformation (Fig. 4G-I). The ALM soma outgrowth phenotype can be rescued by cell-specific expression of $h s f-1$ only in touch neurons (Fig. $4 J)$. HSF-1 transcribes heat shock chaperones, and thus disrupted protein homeostasis might be a critical factor in the production of morphological abnormalities in aging neurons. Since HSF-1 is thought to play a significant role in disaggregation of C. elegans protein aggregates (Cohen et al., 2006), active disaggregation might be an important biochemical activity that protects against generation of morphological abnormalities in aging neurons. The implication of chaperone function in maintenance of architectural integrity suggests a possibility for ageassociated outgrowth initiation-physical blocks in dendrites such as aggregates might induce cytoskeletal rearrangements that result in new outgrowth formation. Regardless of the mechanism operative, the significant activity of HSF-1 in neuroprotection (Pan et al., 2011) is particularly interesting in light of the immense body of data supporting that age-associated protein aggregation promotes neurodegeneration in many late-onset neurodegenerative disorders (Kikis et al., 2010). Our data underscore conclusions from mammalian studies that protein homeostasis is critical for maintaining adult neurons, even in the absence of disease protein stresses.

Finally, it is noteworthy that we have identified a novel phenotype in $h s f-1$ mutant dendrites - a high frequency of persistent broken axons. HSF-1 might thus normally act within the neuron to prevent axon breaking or might be needed to promote repair by rapid refusion of dissociated ends (Neumann et al., 2011); alternatively, HSF-1 might confer system-wide protection via general cell maintenance. We have not found breaks substantial enough to be detected in aging wild-type touch neurons, but breaks in $C$. elegans can occur in some mutant backgrounds $(\beta$ spectrin mutants, for example) (Hammarlund et al., 2007) and repair/regrowth mechanisms have been documented (OrenSuissa et al., 2010; Wang and Jin, 2011).

\section{Synaptic deterioration is prevalent in the aging C. elegans nervous system}

In the aging C. elegans nervous system, synapses appear physically less robust than in younger animals. The average synapse in an elderly animal is smaller in its presynaptic density and includes fewer synaptic vesicles as compared with young adults. Away from the synapse, typical changes in aging neurons include reduced axon caliber, poor axonal transport, smaller nuclei, and reduced cytoplasmic volume. Interestingly, late in life, animals that maintain their vigor better than their same age siblings also better maintain their basic ultrastructural synaptic features, suggestive of higher signaling capacity at these synapses. The parallel effects of synaptic decline and mobility diminution suggest that at least some mobility loss has an origin in nervous system defects, despite the considerable physical deterioration of muscle that constitutes nematode sarcopenia (Herndon et al., 2002). Whether a decline in synaptic integrity can influence novel outgrowth associated with aging remains to be determined, but some C. elegans observations of regrowth in response to genetically induced synaptic dysfunction suggest that this could be the case (Hedgecock et al., 1990; Hall et al., 1997; Zhao and Nonet, 2001; Loria et al., 2004).

Loss of synaptic integrity (Geinisman et al., 1986; Liu et al., 1996; Bourgeois and Ben-Yakar, 2008) and synaptic gene expression (Blalock et al., 2003; Lu et al., 2004) characterizes the aging mammalian brain and might contribute to functional decline (Yankner et al., 2008). C. elegans synaptic gene expression also changes with age (Ch'ng et al., 2008) and our study demonstrates structural alterations in aging C. elegans synapses. Thus, components of age-associated synaptic degeneration appear common across phyla.

\section{C. elegans neuronal aging shares some features of human brain aging}

Aging of the human brain contributes to a decline in specific cognitive functions (for review, see Yankner et al., 2008; Bishop et al., 2010; Fjell and Walhovd, 2010). Healthy brain aging does not include extensive cell death or neuronal loss in most regions tested, but rather includes synaptic deterioration (Loerch et al., 2008), volume loss, and dendritic reorganization. The molecular factors that contribute to this decline are not understood, espe- 
cially at the single cell level. It is striking that aging of the $C$. elegans nervous system involves similar features-little if any cell death, but loss of synaptic integrity and abnormal outgrowths from some neuronal types. Similarities in neuronal aging between nematodes and humans suggest that conserved mechanisms may promote and/or permit decline. Overall, the commonalities of changes in synapses, dendrite restructuring, and insulin modulation of decline between nematodes and mammals support that extending understanding of $C$. elegans neuronal aging may have applications for development of therapeutic interventions that maintain health of the aging human brain.

\section{Notes}

Supplemental material for this article is available at https://sites.google. $\mathrm{com} /$ site/tothneuronalaging/. This material has not been peer reviewed.

\section{References}

Alcedo J, Kenyon C (2004) Regulation of C. elegans longevity by specific gustatory and olfactory neurons. Neuron 41:45-55.

Altun ZF, Herndon A, Crocker C, Lints R, Hall DH (2010) Neurons and circuits. In: WormAtlas. E-book available at www.wormatlas.org.

Antebi A (2007) Genetics of aging in Caenorhabditis elegans. PLoS Genet 3:1565-1571.

Apfeld J, Kenyon C (1999) Regulation of lifespan by sensory perception in Caenorhabditis elegans. Nature 402:804-809.

Bacaj T, Shaham S (2007) Temporal control of cell-specific transgene expression in Caenorhabditis elegans. Genetics 176:2651-2655.

Bishop NA, Lu T, Yankner BA (2010) Neural mechanisms of ageing and cognitive decline. Nature 464:529-535.

Blalock EM, Chen KC, Sharrow K, Herman JP, Porter NM, Foster TC, Landfield PW (2003) Gene microarrays in hippocampal aging: statistical profiling identifies novel processes correlated with cognitive impairment. J Neurosci 23:3807-3819.

Bourgeois F, Ben-Yakar A (2008) Femtosecond laser nanoaxotomy properties and their effect on axonal recovery in C. elegans. Opt Express 16:5963.

Brenner S (1974) The genetics of Caenorhabditis elegans. Genetics 77: 71-94.

Broughton S, Partridge L (2009) Insulin/IGF-like signalling, the central nervous system and aging. Biochem J 418:1-12.

Chalfie M, Thomson JN (1979) Organization of neuronal microtubules in the nematode Caenorhabditis elegans. J Cell Biol 82:278-289.

Chalfie M, Sulston JE, White JG, Southgate E, Thomson JN, Brenner S (1985) The neural circuit for touch sensitivity in Caenorhabditis elegans. J Neurosci 5:956-964.

Chiu SL, Cline HT (2010) Insulin receptor signaling in the development of neuronal structure and function. Neural Dev 5:7.

Ch'ng Q, Sieburth D, Kaplan JM (2008) Profiling synaptic proteins identifies regulators of insulin secretion and lifespan. PLoS Genet 4:e1000283.

Coburn CM, Mori I, Ohshima Y, Bargmann CI (1998) A cyclic nucleotidegated channel inhibits sensory axon outgrowth in larval and adult Caenorhabditis elegans: a distinct pathway for maintenance of sensory axon structure. Development 125:249-258.

Cohen E, Dillin A (2008) The insulin paradox: aging, proteotoxicity and neurodegeneration. Nat Rev Neurosci 9:759-767.

Cohen E, Bieschke J, Perciavalle RM, Kelly JW, Dillin A (2006) Opposing activities protect against age-onset proteotoxicity. Science 313:1604-1610.

Cohen E, Du D, Joyce D, Kapernick EA, Volovik Y, Kelly JW, Dillin A (2010) Temporal requirements of insulin/IGF-1 signaling for proteotoxicity protection. Aging Cell 9:126-134.

Fei YJ, Romero MF, Krause M, Liu JC, Huang W, Ganapathy V, Leibach FH (2000) A novel $\mathrm{H}(+)$-coupled oligopeptide transporter (OPT3) from Caenorhabditis elegans with a predominant function as a $\mathrm{H}(+)$ channel and an exclusive expression in neurons. J Biol Chem 275:9563-9571.

Fjell AM, Walhovd KB (2010) Structural brain changes in aging: courses, causes and cognitive consequences. Rev Neurosci 21:187-221.

Fujimoto M, Nakai A (2010) The heat shock factor family and adaptation to proteotoxic stress. FEBS J 277:4112-4125.

Garigan D, Hsu AL, Fraser AG, Kamath RS, Ahringer J, Kenyon C (2002) Genetic analysis of tissue aging in Caenorhabditis elegans: a role for heatshock factor and bacterial proliferation. Genetics 161:1101-1112.
Geinisman Y, de Toledo-Morrell L, Morrell F (1986) Loss of perforated synapses in the dentate gyrus: morphological substrate of memory deficit in aged rats. Proc Natl Acad Sci U S A 83:3027-3031.

Gerstbrein B, Stamatas G, Kollias N, Driscoll M (2005) In vivo spectrofluorimetry reveals endogenous biomarkers that report healthspan and dietary restriction in Caenorhabditis elegans. Aging Cell 4:127-137.

Glenn CF, Chow DK, David L, Cooke CA, Gami MS, Iser WB, Hanselman KB, Goldberg IG, Wolkow CA (2004) Behavioral deficits during early stages of aging in Caenorhabditis elegans result from locomotory deficits possibly linked to muscle frailty. J Gerontol A Biol Sci Med Sci 59:1251-1260.

Goodman MB (2006) Mechanosensation. WormBook 1-14.

Govorunova EG, Moussaif M, Kullyev A, Nguyen KC, McDonald TV, Hall DH, Sze JY (2010) A homolog of FHM2 is involved in modulation of excitatory neurotransmission by serotonin in C. elegans. PLoS ONE 5:e10368.

Hall DH (1995) Electron microscopy and three-dimensional image reconstruction. Methods Cell Biol 48:395-436.

Hall DH, Gu G, García-Añoveros J, Gong L, Chalfie M, Driscoll M (1997) Neuropathology of degenerative cell death in Caenorhabditis elegans. J Neurosci 17:1033-1045.

Hammarlund M, Jorgensen EM, Bastiani MJ (2007) Axons break in animals lacking beta-spectrin. J Cell Biol 176:269-275.

Hedgecock EM, Culotti JG, Hall DH (1990) The unc-5, unc-6, and unc-40 genes guide circumferential migrations of pioneer axons and mesodermal cells on the epidermis in C. elegans. Neuron 4:61-85.

Herndon LA, Schmeissner PJ, Dudaronek JM, Brown PA, Listner KM, Sakano Y, Paupard MC, Hall DH, Driscoll M (2002) Stochastic and genetic factors influence tissue-specific decline in ageing C. elegans. Nature 419:808-814.

Hsu AL, Murphy CT, Kenyon C (2003) Regulation of aging and age-related disease by DAF-16 and heat-shock factor. Science 300:1142-1145.

Huang C, Xiong C, Kornfeld K (2004) Measurements of age-related changes of physiological processes that predict lifespan of Caenorhabditis elegans. Proc Natl Acad Sci U S A 101:8084-8089.

Ibanez-Ventoso C, Yang M, Guo S, Robins H, Padgett RW, Driscoll M (2006) Modulated microRNA expression during adult lifespan in Caenorhabditis elegans. Aging Cell 5:235-246.

Iser WB, Gami MS, Wolkow CA (2007) Insulin signaling in Caenorhabditis elegans regulates both endocrine-like and cell-autonomous outputs. Dev Biol 303:434-447.

Kenyon CJ (2010) The genetics of ageing. Nature 464:504-512.

Kikis EA, Gidalevitz T, Morimoto RI (2010) Protein homeostasis in models of aging and age-related conformational disease. Adv Exp Med Biol 694:138-159.

Knobloch M, Mansuy IM (2008) Dendritic spine loss and synaptic alterations in Alzheimer's disease. Mol Neurobiol 37:73-82.

Lesa GM, Palfreyman M, Hall DH, Clandinin MT, Rudolph C, Jorgensen EM, Schiavo G (2003) Long chain polyunsaturated fatty acids are required for efficient neurotransmission in C. elegans. J Cell Sci 116:4965-4975.

Liu LX, Spoerke JM, Mulligan EL, Chen J, Reardon B, Westlund B, Sun L, Abel K, Armstrong B, Hardiman G, King J, McCague L, Basson M, Clover R, Johnson CD (1999) High-throughput isolation of Caenorhabditis elegans deletion mutants. Genome Res 9:859-867.

Liu X, Erikson C, Brun A (1996) Cortical synaptic changes and gliosis in normal aging, Alzheimer's disease and frontal lobe degeneration. Dementia 7:128-134.

Loerch PM, Lu T, Dakin KA, Vann JM, Isaacs A, Geula C, Wang J, Pan Y, Gabuzda DH, Li C, Prolla TA, Yankner BA (2008) Evolution of the aging brain transcriptome and synaptic regulation. PLoS ONE 3:e3329.

Loria PM, Hodgkin J, Hobert O (2004) A conserved postsynaptic transmembrane protein affecting neuromuscular signaling in Caenorhabditis elegans. J Neurosci 24:2191-2201.

Lu T, Pan Y, Kao SY, Li C, Kohane I, Chan J, Yankner BA (2004) Gene regulation and DNA damage in the ageing human brain. Nature 429:883-891.

Martin LJ (2010) Mitochondrial and cell death mechanisms in neurodegenerative diseases. Pharmaceuticals 3:839-915.

Marza E, Long T, Saiardi A, Sumakovic M, Eimer S, Hall DH, Lesa GM (2008) Polyunsaturated fatty acids influence synaptojanin localization to regulate synaptic vesicle recycling. Mol Biol Cell 19:833-842.

McIntire SL, Reimer RJ, Schuske K, Edwards RH, Jorgensen EM (1997) 
Identification and characterization of the vesicular GABA transporter. Nature 389:870-876.

Morley JF, Morimoto RI (2004) Regulation of longevity in Caenorhabditis elegans by heat shock factor and molecular chaperones. Mol Biol Cell 15:657-664.

Neumann B, Nguyen KC, Hall, DH, Ben-Yakar A, Hilliard MA (2011) Axonal regeneration proceeds through specific axonal fusion in transected C. elegans neurons. Dev Dyn 240:1365-1372.

Ogg S, Ruvkun G (1998) The C. elegans PTEN homolog, DAF-18, acts in the insulin receptor-like metabolic signaling pathway. Mol Cell 2:887-893.

Oren-Suissa M, Hall DH, Treinin M, Shemer G, Podbilewicz B (2010) The fusogen EFF-1 controls sculpting of mechanosensory dendrites. Science 328:1285-1288

Pan CL, Peng CY, Chen CH, McIntire S (2011) Genetic analysis of agedependent defects of the Caenorhabditis elegans touch receptor neurons. Proc Natl Acad Sci U S A 108:9274-9279.

Panowski SH, Dillin A (2009) Signals of youth: endocrine regulation of aging in Caenorhabditis elegans. Trends Endocrinol Metab 20:259-264.

Peckol EL, Zallen JA, Yarrow JC, Bargmann CI (1999) Sensory activity affects sensory axon development in C. elegans. Development 126:1891-1902.

Rea SL, Wu D, Cypser JR, Vaupel JW, Johnson TE (2005) A stress-sensitive reporter predicts longevity in isogenic populations of Caenorhabditis elegans. Nat Genet 37:894-898.

Sawin ER, Ranganathan R, Horvitz HR (2000) C. elegans locomotory rate is modulated by the environment through a dopaminergic pathway and by experience through a serotonergic pathway. Neuron 26:619-631.

Suh Y, Atzmon G, Cho MO, Hwang D, Liu B, Leahy DJ, Barzilai N, Cohen P (2008) Functionally significant insulin-like growth factor I receptor mutations in centenarians. Proc Natl Acad Sci U S A 105:3438-3442.

Sulston J, Dew M, Brenner S (1975) Dopaminergic neurons in the nematode Caenorhabditis elegans. J Comp Neurol 163:215-226.

Taguchi A, Wartschow LM, White MF (2007) Brain IRS2 signaling coordinates life span and nutrient homeostasis. Science 317:369-372.

Tank EM, Rodgers KE, Kenyon C (2011) Spontaneous Age-Related Neurite Branching in Caenorhabditis elegans. J Neurosci 31:9279-9288.

Wang Z, Jin Y (2011) Genetic dissection of axon regeneration. Curr Opin Neurobiol 21:189-196.

White JG, Southgate E, Thomson JN, Brenner S (1986) The structure of the nervous system of the nematode Caenorhabditis elegans. Philos Trans R Soc Lond B Biol Sci 314:1-340.

Wolkow CA, Kimura KD, Lee MS, Ruvkun G (2000) Regulation of C. elegans life-span by insulinlike signaling in the nervous system. Science 290:147-150.

Yankner BA, Lu T, Loerch P (2008) The aging brain. Annu Rev Pathol 3:41-66.

Zhao H, Nonet ML (2001) A conserved mechanism of synaptogyrin localization. Mol Biol Cell 12:2275-2289. 